\begin{tabular}{|c|l|}
\hline Title & Spacelike surfaces in Anti de Sitter four-space from a contact view point \\
\hline Author(s) & Izumiya, Shyuichi; Pei, Donghe; Romero Fuster, Maria del Carmen \\
\hline Citation & Hokkaido University Preprint Series in Mathematics, 914, 1-20 \\
\hline Issue Date & 2008-05-07 \\
\hline DOI & 10.14943/84063 \\
\hline Doc URL & http://hdl.handle.net/2115/69721 \\
\hline Type & bulletin (article) \\
\hline File Information & pre914.pdf \\
\hline
\end{tabular}

Instructions for use 


\title{
Spacelike surfaces in Anti de Sitter four-space from a contact viewpoint
}

\author{
Shyuichi Izumiya, Donghe Pei *and María del Carmen Romero Fuster ${ }^{\dagger}$
}

May 7, 2008

Dedicated to Vladimir Igorevich Arnold on the occasion of his 70th birthday

\begin{abstract}
We define the notions of $S_{t}^{1} \times S_{s}^{2}$-nullcone Legendrian Gauss maps and $S_{+}^{2}$-nullcone Lagrangian Gauss maps on spacelike surfaces in Anti de Sitter 4-space. We investigate the relationships between singularities of these maps and geometric properties of surfaces as an application of the theory of Legendrian/Lagrangian singularities. By using $S_{+}^{2}$-nullcone Lagrangian Gauss maps, we define the notion of $S_{+}^{2}$-nullcone Gauss-Kronecker curvatures and show a Gauss-Bonnet type theorem as a global property. We also introduce the notion of horospherical Gauss maps whch has different geometric properties of the above Gauss maps. As a consequence, we can say that Anti de Sitter space has much more rich geometric properties than the other space forms such as Euclidean space, Hyperbolic space, Lorentz-Minkowski space and de Sitter space.
\end{abstract}

\section{Introduction}

The study of Anti de Sitter 4-space is of special interested in the theory of relativity, for it represents one of the vacuum solutions of the Einstein equation. We observe that it is a Lorentzian space form with negative curvature. It is well-known that the Lorentzian space form with zero curvature is Lorentz-Minkowski space and with positive curvature is de Sitter space. These Lorentzian space forms have been well studied (cf., $[10,11,13,14,15,16]$ ). However, there are not much results on submanifolds immersed in Anti de Sitter space, in particular from the viewpoint of singularity theory. We must remark that although Anti de Sitter space is diffeomorphic to de Sitter space, their causalities (i.e., the structure as a Lorentzian manifold) are quite different.

\footnotetext{
*Work partially supported by NSF of China, grant No. 10471020 and NCET of China, grant No. 05-0319.

†Work partially supported by DGCYT grant no. BMF2006-02037 and FEDER.

2000 Mathematics Subject classification. Primary 53A35; Secondary 58C27

Key Words and Phrases. Anti de Sitter 4-space, $S_{t}^{1} \times S_{s}^{2}$-nullcone Legendrian Gauss map, $S_{+}^{2}$-nullcone Lagrangian Gauss map, lightlike hyperbolic cylinder.
} 
In the study of submanifolds in Lorentzian-Minkowski space, the codimension two spacelike submanifolds happen to be the most interesting subjects, both from the view point of singularity theory and of the theory of relativity (cf., $[10,11,13,14,15,16]$ ). For instance, one of the important objects in the theory of relativity are the lightlike hypersurfaces because they provide good models for different types of horizons [6, 20]. A lightlike hypersurface is a ruled hypersurface along a spacelike surface whose rulings are lightlike geodesics. This is one of the motivations for studying the spacelike surfaces in a 4-dimensional Lorentzian space form. In [10] we studied singularities of lightcone Gauss maps of spacelike surface in Minkowski 4-space, and established the relationships between such singularities and geometric invariants of these surfaces under the action of Lorentz group. Our aim in this paper is to develop the analogous study for the spacelike surfaces in Anti de Sitter 4-space. For this purpose, we shall adapt the tools developed in the previous paper for the study of spacelike surfaces in Minkowski space to that of the spacelike surfaces in Anti de Sitter 4-space. To do this we need to develop first the local differential geometry of spacelike surface in Anti de Sitter 4-space in a similar way as the classically done for surfaces in Euclidean 4-space [17] and Lorentz-Minkowski 4-space [10]. As it was to be expected, the situation presents certain peculiarities when compared with the Euclidean case and Lorentz-Minkowski case. For instance, in our case it is always possible to choose two lightlike normal directions along the spacelike surface in a frame of its normal bundle. This is similar to the Lorentz-Minkowski case, but the image is located in three dimensional space $S_{t}^{1} \times S_{s}^{2}$. For the Lorentz-Mikowski case, the image of the lightcone Gauss map is located in the three dimensional spacelike sphere $S_{+}^{3}$. By using this, we define the $S_{t}^{1} \times S_{s}^{2}$-nullcone Legendrian Gauss map and the normalized lightlike (null) Gauss-Kronecker curvature $\widetilde{\mathcal{K}}_{\ell}(1, \pm 1)$ of the spacelike surface in Anti de Sitter 4 -space. We introduce the notion of nullcone height function and use it to show that the $S_{t}^{1} \times S_{s}^{2}$-nullcone Legendrian Gauss map has a singular point if and only if the lightlike Gauss-Kronecker curvature vanishes at such point. Moreover, we show that the $S_{t}^{1} \times S_{s}^{2}$-nullcone Legendrian Gauss map is a constant map if and only if the spacelike surface is contained in the intersection of a lightlike hyperplane and Anti de Sitter 4-space which we call a lightlike hyperbolic cylinder in Anti de Sitter space, so that we can view the singularities of the $S_{t}^{1} \times S_{s}^{2}$-nullcone Legendrian Gauss map as an estimate of the contacts of the surface with lightlike hyperbolic cylinders.

On the other hand, we introduce a natural $S^{1}$-fibration, $\tau: A d S^{4} \rightarrow H_{+}^{3}(-1)$. This fibration induces the $S_{+}^{2}$-nullcone Lagrangian Gauss map and the $S_{+}^{2}$-nullcone Gauss-Kronecker curvature of the spacelike surface. We show that $S_{t}^{1} \times S_{s}^{2}$-nullcone Legendrian Gauss map is a Legendrian covering of $S_{+}^{2}$-nullcone Lagrangian Gauss map, so that the singularities of these mappings (the parabolic points with respect to the corresponding curvatures) are the same. Moreover, we show that the Gauss-Bonnet type theorem for $S_{+}^{2}$-nullcone Gauss-Kronecker curvature as a global property.

We also define a horospherical Gauss map together with its horospherical height functions on the spacelike surfaces in Anti de Sitter 4-space as an application of the horospherical geometry in Hyperbolic space[9, 12]. Such functions measure the contacts of the surface with certain $S O(2) \times S O(3)$ invariant submanifolds that we call here round horospheres, where $S O(2) \times S O(3)$ is canonically embedded subgroup of the group of semi-Euclidean motions $S O(2,3)$. These are obtained as the pull-back by of the horospheres of $H_{+}^{3}(-1)$ by $\widehat{\tau}$. This Gauss map has an associated horospherical Gauss-Kronecker curvature as well as principal configurations. We state some global properties concerning these, obtained by pull-back of the corresponding results on surfaces in $H_{+}^{3}(-1)([12,14])$. 
We shall assume throughout the whole paper that all the maps and manifolds are $C^{\infty}$ unless the contrary is explicitly stated.

\section{Local differential geometry on spacelike surfaces in Anti de Sitter 4-space}

Let $\mathbb{R}^{5}=\left\{\left(x_{1}, \ldots, x_{5}\right) \mid x_{i} \in \mathbb{R}(i=1, \ldots, 5)\right\}$ be a 5 -dimensional vector space. For any vectors $\boldsymbol{x}=\left(x_{1}, \ldots, x_{5}\right)$ and $\boldsymbol{y}=\left(y_{1}, \ldots, y_{5}\right)$ in $\mathbb{R}^{5}$, the pseudo scalar product of $\boldsymbol{x}$ and $\boldsymbol{y}$ is defined to be $\langle\boldsymbol{x}, \boldsymbol{y}\rangle=-x_{1} y_{1}-x_{2} y_{2}+\sum_{i=3}^{5} x_{i} y_{i}$. We call $\left(\mathbb{R}^{5},\langle\rangle,\right)$ a semi-Euclidean 5-space with index 2 and write $\mathbb{R}_{2}^{5}$ instead of $\left(\mathbb{R}^{5},\langle\rangle,\right)$.

We say that a vector $\boldsymbol{x}$ in $\mathbb{R}_{2}^{5} \backslash\{\mathbf{0}\}$ is spacelike, null or timelike if $\langle\boldsymbol{x}, \boldsymbol{x}\rangle>0,=0$ or $<0$ respectively. The norm of the vector $\boldsymbol{x} \in \mathbb{R}_{2}^{5}$ is defined by $\|\boldsymbol{x}\|=\sqrt{|\langle\boldsymbol{x}, \boldsymbol{x}\rangle|}$. For a null vector $\boldsymbol{n} \in \mathbb{R}_{2}^{5}$ and a real number $c$, we define the null hyperplane with pseudo normal $\boldsymbol{n}$ by

$$
N H(\boldsymbol{n}, c)=\left\{\boldsymbol{x} \in \mathbb{R}_{2}^{5} \mid\langle\boldsymbol{x}, \boldsymbol{n}\rangle=c\right\} .
$$

We now define Anti de Sitter 4-space by

$$
A d S^{4}=\left\{\boldsymbol{x} \in \mathbb{R}_{2}^{5} \mid\langle\boldsymbol{x}, \boldsymbol{x}\rangle=-1\right\} .
$$

We also define

$$
\Lambda_{p}=\left\{\boldsymbol{x}=\left(x_{1}, \ldots, x_{5}\right) \in \mathbb{R}_{2}^{5} \mid-\left(x_{1}-p_{1}\right)^{2}-\left(x_{2}-p_{2}\right)^{2}+\sum_{i=3}^{5}\left(x_{i}-p_{i}\right)^{2}=0\right\}
$$

and

$$
S_{t}^{1} \times S_{s}^{2}=\left\{\boldsymbol{x}=\left(x_{1}, \ldots, x_{5}\right) \in \Lambda:=\Lambda_{0}^{*} \mid x_{1}^{2}+x_{2}^{2}=1\right\},
$$

where $p=\left(p_{1}, \ldots, p_{5}\right) \in \mathbb{R}_{2}^{5}, S_{t}^{1}$ denotes the timelike circle and $S_{s}^{2}$ denotes the spacelike sphere. We call $\Lambda_{p}^{*}=\Lambda_{p} \backslash\{p\}$ the nullcone at the vertex $p$. Given any null vector $\boldsymbol{x}=\left(x_{1}, \ldots, x_{5}\right)$, we have

$$
\overline{\boldsymbol{x}}=\left(\frac{x_{1}}{\sqrt{x_{1}^{2}+x_{2}^{2}}}, \frac{x_{2}}{\sqrt{x_{1}^{2}+x_{2}^{2}}}, \frac{x_{3}}{\sqrt{x_{1}^{2}+x_{2}^{2}}}, \frac{x_{4}}{\sqrt{x_{1}^{2}+x_{2}^{2}}}, \frac{x_{5}}{\sqrt{x_{1}^{2}+x_{2}^{2}}}\right) \in S_{t}^{1} \times S_{s}^{2} .
$$

It is known that Anti de Sitter 4-space is time orientable, so that we fix a time orientation of Anti de Sitter 4-space. Therefore we can distinguish that any timelike vector has the future direction or the past direction.

On the other hand, we study the local differential geometry of spacelike surface in $A d S^{4}$. Let $\boldsymbol{X}: U \rightarrow A d S^{4}$ be an embedding from an open subset $U \subset \mathbb{R}^{2}$. We denote that $M=\boldsymbol{X}(U)$ and identify $M$ and $U$ through the embedding $\boldsymbol{X}$. We say that $M$ is a spacelike surface if the tangent space $T_{p} M$ of $M$ is a spacelike plane in Anti de Sitter 4-space for any point $p \in M$. In this case, the normal space $N_{p} M$ is a Lorentzian plane in Anti de Sitter 4space(cf.,[23]). Let $\left\{\boldsymbol{e}_{3}(p), \boldsymbol{e}_{4}(p) ; p=\boldsymbol{X}(x, y)\right\}$ be an orthonormal frame of the tangent space $T_{p} M$ and $\left\{\boldsymbol{e}_{1}(p), \boldsymbol{e}_{2}(p) ; p\right\}$ a pseudo-orthonormal frame of $N_{p} M$ in Anti de Sitter 4-space, where, $\boldsymbol{e}_{1}(p)$ are unit timelike vectors and $\boldsymbol{e}_{2}(p), \boldsymbol{e}_{3}(p), \boldsymbol{e}_{4}(p)$ are unit spacelike vectors. Then $\left\{\boldsymbol{e}_{1}(p), \boldsymbol{e}_{2}(p), \boldsymbol{e}_{3}(p), \boldsymbol{e}_{4}(p), \boldsymbol{e}_{5}(p)=\boldsymbol{p}=\boldsymbol{X}(p) ; p\right\}$ is a pseudo-orthonormal frame of $\mathbb{R}_{2}^{5}$ at $p$.

We shall now establish the fundamental formula for a spacelike surface in Anti de Sitter 4-space by means of similar notions to those of Little [17]. 
We can write $d \boldsymbol{X}=\sum_{i=1}^{5} \omega_{i} \boldsymbol{e}_{i}$ and $d \boldsymbol{e}_{i}=\sum_{j=1}^{5} \omega_{i j} \boldsymbol{e}_{j} ; i=1, \ldots, 5$. where $\omega_{i}$ and $\omega_{i j}$ are 1 -forms given by $\omega_{i}=\delta\left(\boldsymbol{e}_{i}\right)\left\langle d \boldsymbol{X}, \boldsymbol{e}_{i}\right\rangle$ and $\omega_{i j}=\delta\left(\boldsymbol{e}_{j}\right)\left\langle d \boldsymbol{e}_{i}, \boldsymbol{e}_{j}\right\rangle$, whith

$$
\delta\left(\boldsymbol{e}_{i}\right)=\operatorname{sign}\left(\boldsymbol{e}_{i}\right)= \begin{cases}1 & i=2,3,4 \\ -1 & i=1,5 .\end{cases}
$$

We have the Codazzi type equations:

$$
\left\{\begin{array}{l}
d \omega_{i}=\sum_{j=1}^{5} \delta\left(\boldsymbol{e}_{i}\right) \delta\left(\boldsymbol{e}_{j}\right) \omega_{i j} \wedge \omega_{j} \\
d \omega_{i j}=\sum_{k=1}^{5} \omega_{i k} \wedge \omega_{k j},
\end{array}\right.
$$

where $d$ is exterior derivative.

Since $\left\langle\boldsymbol{e}_{i}, \boldsymbol{e}_{j}\right\rangle=\delta_{i j} \delta\left(\boldsymbol{e}_{j}\right)$ (where $\delta_{i j}$ is Kronecker's delta), we get

$$
\omega_{i j}=-\delta\left(\boldsymbol{e}_{i}\right) \delta\left(\boldsymbol{e}_{j}\right) \omega_{j i} .
$$

In particular, $\omega_{i i}=0 ; i=1, \ldots, 5$. It follows from the fact $\left\langle d \boldsymbol{X}, \boldsymbol{e}_{1}\right\rangle=\left\langle d \boldsymbol{X}, \boldsymbol{e}_{2}\right\rangle=\langle d \boldsymbol{X}, \boldsymbol{X}\rangle=0$ that

$$
\omega_{1}=\omega_{2}=\omega_{5}=0
$$

Therefore we have

$$
\left\{\begin{array}{l}
0=d \omega_{1}=\sum_{j=1}^{4} \delta\left(\boldsymbol{e}_{1}\right) \delta\left(\boldsymbol{e}_{j}\right) \omega_{1 j} \wedge \omega_{j}=\sum_{j=3}^{4} \delta\left(\boldsymbol{e}_{1}\right) \delta\left(\boldsymbol{e}_{j}\right) \omega_{1 j} \wedge \omega_{j}=-\omega_{13} \wedge \omega_{3}-\omega_{14} \wedge \omega_{4}, \\
0=d \omega_{2}=\sum_{j=1}^{4} \delta\left(\boldsymbol{e}_{2}\right) \delta\left(\boldsymbol{e}_{j}\right) \omega_{2 j} \wedge \omega_{j}=\sum_{j=3}^{4} \delta\left(\boldsymbol{e}_{2}\right) \delta\left(\boldsymbol{e}_{j}\right) \omega_{2 j} \wedge \omega_{j}=\omega_{23} \wedge \omega_{3}+\omega_{24} \wedge \omega_{4}, \\
0=d \omega_{5}=\sum_{j=1}^{4} \delta\left(\boldsymbol{e}_{5}\right) \delta\left(\boldsymbol{e}_{j}\right) \omega_{5 j} \wedge \omega_{j}=\sum_{j=3}^{4} \delta\left(\boldsymbol{e}_{5}\right) \delta\left(\boldsymbol{e}_{j}\right) \omega_{5 j} \wedge \omega_{j}=-\omega_{35} \wedge \omega_{3}-\omega_{45} \wedge \omega_{4} .
\end{array}\right.
$$

By Cartan's lemma, we can write

$$
\left\{\begin{array}{l}
\omega_{13}=a \omega_{3}+b \omega_{4}, \omega_{14}=b \omega_{3}+c \omega_{4}, \\
\omega_{23}=e \omega_{3}+f \omega_{4}, \omega_{24}=f \omega_{3}+g \omega_{4}, \\
\omega_{35}=h \omega_{3}+m \omega_{4}, \omega_{45}=m \omega_{3}+n \omega_{4} .
\end{array}\right.
$$

for appropriate functions $a, b, c, e, f, g, h, m$ and $n$.

We define $\left\langle d^{2} \boldsymbol{X}, \boldsymbol{e}_{i}\right\rangle=-\left\langle d \boldsymbol{X}, d \boldsymbol{e}_{i}\right\rangle, i=1,2,5$, from which we get a vector-valued quadratic form:

$-\left\langle d^{2} \boldsymbol{X}, \boldsymbol{e}_{1}\right\rangle \boldsymbol{e}_{1}+\left\langle d^{2} \boldsymbol{X}, e_{2}\right\rangle \boldsymbol{e}_{2}-\left\langle d^{2} \boldsymbol{X}, \boldsymbol{e}_{5}\right\rangle \boldsymbol{e}_{5}=\left(a \omega_{3}^{2}+2 b \omega_{3} \omega_{4}+c \omega_{4}^{2}\right) \boldsymbol{e}_{1}-\left(e \omega_{3}^{2}+2 f \omega_{3} \omega_{4}+g \omega_{4}^{2}\right) \boldsymbol{e}_{2}+\left(\omega_{3}^{2}+\omega_{4}^{2}\right) \boldsymbol{e}_{5}$, which is called the second fundamental form of the spacelike surface in $\mathbb{R}_{2}^{5}$. It follows from (2) that

$$
d\left(\begin{array}{c}
\boldsymbol{e}_{1}+\boldsymbol{e}_{2} \\
\boldsymbol{e}_{1}-\boldsymbol{e}_{2} \\
\boldsymbol{e}_{3} \\
\boldsymbol{e}_{4} \\
\boldsymbol{e}_{5}
\end{array}\right)=\left(\begin{array}{ccccc}
0 & \omega_{12} & \omega_{13}+\omega_{23} & \omega_{14}+\omega_{24} & 0 \\
-\omega_{12} & 0 & \omega_{13}-\omega_{23} & \omega_{14}-\omega_{24} & 0 \\
\frac{\omega_{13}+\omega_{23}}{2} & \frac{\omega_{13}-\omega_{23}}{2} & 0 & \omega_{34} & \omega_{35} \\
\frac{\omega_{14}+\omega_{24}}{2} & \frac{\omega_{14}-\omega_{24}}{2} & -\omega_{34} & 0 & \omega_{45} \\
0 & 0 & \omega_{35} & \omega_{45} & 0
\end{array}\right)\left(\begin{array}{c}
\boldsymbol{e}_{1}-\boldsymbol{e}_{2} \\
\boldsymbol{e}_{1}+\boldsymbol{e}_{2} \\
\boldsymbol{e}_{3} \\
\boldsymbol{e}_{4} \\
\boldsymbol{e}_{5}
\end{array}\right) .
$$


Let $\boldsymbol{e}_{1}=\left(a_{1}, \ldots, a_{5}\right), \boldsymbol{e}_{2}=\left(b_{1}, \ldots, b_{5}\right), \xi^{ \pm}=\sqrt{\left(a_{1}-b_{1}\right)^{2} \pm\left(a_{2}-b_{2}\right)^{2}}$, then we have the following fundamental formula:

$$
d\left(\begin{array}{c}
\overline{\boldsymbol{e}_{1}+\boldsymbol{e}_{2}} \\
\hline \boldsymbol{e}_{1}-\boldsymbol{e}_{2} \\
\boldsymbol{e}_{3} \\
\boldsymbol{e}_{4} \\
\boldsymbol{e}_{5}
\end{array}\right)=\left(\begin{array}{ccccc}
0 & \omega_{12}-\frac{d \xi^{+}}{\xi^{+}} & \frac{\omega_{13}+\omega_{23}}{\xi^{+}} & \frac{\omega_{14}+\omega_{24}}{\xi^{+}} & 0 \\
-\omega_{12}-\frac{d \xi^{-}}{\xi^{-}} & 0 & \frac{\omega_{13}-\omega_{23}}{\xi^{-}} & \frac{\omega_{14}-\omega_{24}}{\xi^{-}} & 0 \\
\frac{\omega_{13}+\omega_{23}}{2} & \frac{\omega_{13}-\omega_{23}}{2} & 0 & \omega_{34} & \omega_{35} \\
\frac{\omega_{14}+\omega_{24}}{2} & \frac{\omega_{14}-\omega_{24}}{2} & -\omega_{34} & 0 & \omega_{45} \\
0 & 0 & \omega_{35} & \omega_{45} & 0
\end{array}\right)\left(\begin{array}{c}
\overline{\boldsymbol{e}_{1}-\boldsymbol{e}_{2}} \\
\overline{\boldsymbol{e}_{1}+\boldsymbol{e}_{2}} \\
\boldsymbol{e}_{3} \\
\boldsymbol{e}_{4} \\
\boldsymbol{e}_{5}
\end{array}\right)
$$

Given $\boldsymbol{v}=\xi \boldsymbol{e}_{1}+\eta \boldsymbol{e}_{2} \in N_{p} M$ in $A d S^{4}$, we have $d \boldsymbol{v}=d \xi \boldsymbol{e}_{1}+\xi d \boldsymbol{e}_{1}+d \eta \boldsymbol{e}_{2}+\eta d \boldsymbol{e}_{2}$, and

$$
\left\langle d \boldsymbol{v}, \boldsymbol{e}_{3}\right\rangle \wedge\left\langle d \boldsymbol{v}, \boldsymbol{e}_{4}\right\rangle=\mathcal{K}_{\ell}(\xi, \eta) \omega_{3} \wedge \omega_{4},
$$

where the function $\mathcal{K}_{\ell}$ is given by:

$$
\mathcal{K}_{\ell}(\xi, \eta)=(a \xi \pm e \eta)(c \xi \pm g \eta)-(b \xi+f \eta)^{2}
$$

The function $\mathcal{K}_{\ell}(1, \pm 1$ ) is called the lightlike (or, null) Gauss-Kornecker curvature of $M=$ $\boldsymbol{X}(U)$. We also have a function

$$
\overline{\mathcal{K}}_{\ell}^{ \pm}(p)=\mathcal{K}_{\ell}\left(\frac{1}{\xi^{ \pm}(p)}, \frac{ \pm 1}{\xi^{ \pm}(p)}\right)(p)=\frac{1}{\left(\xi^{ \pm}\right)^{2}(p)} \mathcal{K}_{\ell}(1, \pm 1)(p)
$$

We call $\overline{\mathcal{K}}_{\ell}^{ \pm}$the normalized lightlike Gauss-Kronecker curvature of $M=\boldsymbol{X}(U)$.

On the other hand, we choose the frame $\boldsymbol{e}_{3}, \boldsymbol{e}_{4}$ of $T_{p} M$ with the same orientation as the frame $\boldsymbol{X}_{x}, \boldsymbol{X}_{y}$. We also choose $\boldsymbol{e}_{1}$ as the future directed unit normal timelike vector field. Then we can take the spacelike unit vector $\boldsymbol{e}_{2} \operatorname{such}$ that $\operatorname{det}\left(\boldsymbol{e}_{1}, \boldsymbol{e}_{2}, \boldsymbol{e}_{3}, \boldsymbol{e}_{4}, \boldsymbol{e}_{5}\right)>0$. If we have another future directed unit normal timelike vector field $\boldsymbol{e}_{1}^{\prime}$, we can choose another $\boldsymbol{e}_{2}^{\prime}$. However, since the normal space of $M$ in $A d S^{4}$ is a Lorentzian plane, we have $\overline{\boldsymbol{e}_{1} \pm \boldsymbol{e}_{2}}=\overline{\boldsymbol{e}_{1}^{\prime} \pm \boldsymbol{e}_{2}^{\prime}}$. Therefore we have a well defined map, $\mathbb{N G}_{M}^{ \pm}: M \longrightarrow S_{t}^{1} \times S_{s}^{2}$ given by $\mathbb{N G}_{M}^{ \pm}(p)=\overline{\boldsymbol{e}_{1} \pm \boldsymbol{e}_{2}}(p)$. We call it the $S_{t}^{1} \times S_{s}^{2}$-nullcone Legendrian Gauss map of $M=\boldsymbol{X}(U)$. By the above construction, we have

$$
\overline{\mathcal{K}}_{\ell}^{ \pm}(p)=\operatorname{det}\left(\pi_{p}^{t} \circ d \mathbb{N} \mathbb{G}_{M}^{ \pm}\right)(p),
$$

where $\pi^{t}: T_{p} \mathbb{R}_{2}^{5}=T_{p} M \oplus \bar{N}_{p}(M) \longrightarrow T_{p} M$ is the canonical projection onto the tangent space of $M$. We call $\pi_{p}^{t} \circ d \mathbb{N}_{M}^{ \pm}(p)$ the normalized lightlike Weingarten map of $M$ at $p$.

On the other hand, we consider a projection $\tau: \mathbb{R}^{2} \backslash\{\mathbf{0}\} \rightarrow \mathbb{R}$ defined by $\tau(\lambda \cos \theta, \lambda \sin \theta)=$ $\lambda$ for $(\lambda \cos \theta, \lambda \sin \theta) \in \mathbb{R}^{2} \backslash\{0\}$. This induces a projection, $\widehat{\tau}: \mathbb{R}_{2 *}^{5} \rightarrow \mathbb{R}_{1+}^{4}$ defined by $\widehat{\tau}\left(x_{1}, x_{2}, x_{3}, x_{4}, x_{5}\right)=\left(0, \tau\left(x_{1}, x_{2}\right), x_{3}, x_{4}, x_{5}\right)$, where

$$
\begin{aligned}
\mathbb{R}_{2 *}^{5} & =\left\{\boldsymbol{x}=\left(x_{1}, x_{2}, x_{3}, x_{4}, x_{5}\right) \in \mathbb{R}_{2}^{5} \mid\left(x_{1}, x_{2}\right) \neq(0,0)\right\} \\
\mathbb{R}_{1+}^{4} & =\left\{\boldsymbol{x}=\left(x_{1}, x_{2}, x_{3}, x_{4}, x_{5}\right) \in \mathbb{R}_{2}^{5} \mid x_{1}=0, x_{2}>0\right\} .
\end{aligned}
$$

We can easily show that $\widehat{\tau}$ satisfies $\langle\tau(\boldsymbol{x}), \tau(\boldsymbol{x})\rangle_{1}=\langle\boldsymbol{x}, \boldsymbol{x}\rangle_{2}$, for all $\boldsymbol{x} \in \mathbb{R}_{2 *}^{5}$. That is, it preserves the pseudo-norm of the vectors $\boldsymbol{x} \in \mathbb{R}_{2 *}^{5}$. Consequently it transforms timelike, spacelike and lightlike vectors of $\mathbb{R}_{2 *}^{5}$ respectively, into timelike, spacelike and lightlike vectors in $\mathbb{R}_{1+}^{4}$. Moreover, it determines a $S^{1}$-fibration, $\widehat{\tau}: A d S^{4} \rightarrow H_{+}^{3}(-1)$, since $\left(\lambda \cos \theta, \lambda \sin \theta, x_{3}, x_{4}, x_{5}\right) \in \operatorname{Ad} S^{4}$, 
we have that $-\lambda^{2}+x_{3}^{2}+x_{4}^{2}+x_{5}^{2}=-1$, and thus $\left(0, \lambda, x_{3}, x_{4}, x_{5}\right) \in H_{+}^{3}(-1)$. Here we consider $H_{+}^{3}(-1)=\left\{\left(0, x_{2}, x_{3}, x_{4}, x_{5}\right) \in \mathbb{R}_{1+}^{4} \mid-x_{2}^{2}+x_{3}^{2}+x_{4}^{2}+x_{5}^{2}=-1\right\}$. We also have that $\widehat{\tau}\left(S_{t}^{1} \times S_{s}^{2}\right)=\left\{\boldsymbol{x} \in \mathbb{R}_{1+}^{4} \mid\langle\boldsymbol{x}, \boldsymbol{x}\rangle=0, x_{2}=1\right\}=S_{+}^{2}$ (lightcone unit 2-sphere in $\left.\mathbb{R}_{1}^{4}\right)$. The mapping $\widehat{\tau} \mid S_{t}^{1} \times S_{s}^{2}: S_{t}^{1} \times S_{s}^{2} \longrightarrow S_{+}^{2}$ defines the $S^{1}$-fibration structure over $S_{+}^{2}$. By using these $S^{1}$-fibrations, we we define a map $\widehat{\mathbb{N G}}_{M}^{ \pm}: U \longrightarrow S_{+}^{2}$ by $\widehat{\mathbb{N G}}_{M}^{ \pm}(p)=\widehat{\tau} \circ \mathbb{N} \mathbb{G}_{M}^{ \pm}(p)$, which is called the $S_{+}^{2}$-nullcome Lagrangian Gauss map of $M=\boldsymbol{X}(U)$. By taking the derivative of the $S_{+}^{2}$-nullcome Lagrangian Gauss map, we can define the $S_{+}^{2}$-nullcone Gauss-Kronecker curvature $\widehat{\mathcal{K}}_{\ell}^{ \pm}$by

$$
\left\langle\widehat{\mathbb{N G}}_{M}^{ \pm}, \boldsymbol{e}_{3}\right\rangle \wedge\left\langle d \widehat{\mathbb{N G}}_{M}^{ \pm}, \boldsymbol{e}_{4}\right\rangle=\widehat{\mathcal{K}}_{\ell}^{ \pm} \omega_{3} \wedge \omega_{4}
$$

It follows that

$$
\widehat{\mathcal{K}}_{\ell}^{ \pm}(p)=\operatorname{det}\left(\pi_{p}^{t} \circ d \widehat{\mathbb{N G}}_{M}^{ \pm}\right)(p) .
$$

We respectively denote the Riemannian metrics $g_{M}$ and $g_{S_{+}^{2}}$ on $M$ and $S_{+}^{2}$ which are induced from the semi-Euclidean scalar product $\langle$,$\rangle . Moreover, we denote the area forms on M$ and $S_{+}^{2}$ by $d \mathfrak{a}_{M}$ and $d \mathfrak{a}_{S_{+}^{2}}$ respectively. By definition, we have

$$
\left(\widehat{\mathbb{N G}}_{M}^{ \pm}\right)^{*} d \mathfrak{a}_{S_{+}^{2}}=\widehat{\mathcal{K}}_{\ell}^{ \pm} d \mathfrak{a}_{M}
$$

\section{Nullcone height functions}

In this section we introduce the notion of nullcone height functions on spacelike surfaces in $A d S^{4}$ which is useful for the study of singularities of $S_{t}^{1} \times S_{s}^{2}$-nullcone Legendrian Gauss maps. Given a spacelike surface $M(=\boldsymbol{X}(U)) \subset A d S^{4} \subset \mathbb{R}_{2}^{5}$, we define a function $H: U \times S_{t}^{1} \times S_{s}^{2} \longrightarrow \mathbb{R}$ by $H((x, y), \boldsymbol{w})=\langle\boldsymbol{X}(x, y), \boldsymbol{w}\rangle$, where $\boldsymbol{w}=\left(\sin \theta, \cos \theta, w_{3}, w_{4}, w_{5}\right) \in S_{t}^{1} \times S_{s}^{2}, 0 \leq \theta<\pi$. We call $H$ the nullcone height function on the spacelike surface $M$. We denote $h_{w_{0}}(x, y)=H\left(x, y, \boldsymbol{w}_{0}\right)$, for any fixed $\boldsymbol{w}_{0} \in S_{t}^{1} \times S_{s}^{2}$. Then we have the following proposition.

Proposition 3.1 Let $M$ be a spacelike surface in $A d S^{4} \subset \mathbb{R}_{2}^{5}$ and $H: U \times S_{t}^{1} \times S_{s}^{2} \longrightarrow \mathbb{R} a$ nullcone height function. Then we have the following assertions:

(1) $h_{w}\left(p_{0}\right)=\left(\partial h_{w} / \partial x\right)\left(p_{0}\right)=\left(\partial h_{w} / \partial y\right)\left(p_{0}\right)=0$ if and only if $\boldsymbol{w}=\mu\left(\boldsymbol{e}_{1} \pm \boldsymbol{e}_{2}\right)\left(p_{0}\right)=\overline{\boldsymbol{e}_{1} \pm \boldsymbol{e}_{2}}\left(p_{0}\right)$, where

$\boldsymbol{e}_{1}\left(p_{0}\right)=\left(a_{1}, \ldots, a_{5}\right), \boldsymbol{e}_{2}\left(p_{0}\right)=\left(b_{1}, \ldots, b_{5}\right), \mu=\frac{1}{\sqrt{\left(a_{1} \pm b_{1}\right)^{2}+\left(a_{2} \pm b_{2}\right)^{2}}}$ and $p_{0}=\left(x_{0}, y_{0}\right) \in M$.

(2) $h_{w}\left(p_{0}\right)=\left(\partial h_{w} / \partial x\right)\left(p_{0}\right)=\left(\partial h_{w} / \partial y\right)\left(p_{0}\right)=\operatorname{det} \mathcal{H}\left(h_{w}\right)\left(p_{0}\right)=0$ if and only if $\boldsymbol{w}=\overline{\boldsymbol{e}_{1} \pm \boldsymbol{e}_{2}}\left(p_{0}\right)$ and $\mathcal{K}_{\ell}(1, \pm 1)\left(p_{0}\right)=0$. Here, $\operatorname{det} \mathcal{H}\left(h_{w}\right)\left(p_{0}\right)$ is the determinant of the Hessian matrix of $h_{w}$ at $p_{0}$.

Proof. By a straight forward calculation, $h_{w}\left(p_{0}\right)=\left(\partial h_{w} / \partial x\right)\left(p_{0}\right)=\left(\partial h_{w} / \partial y\right)\left(p_{0}\right)=0$ if and only if

$$
\langle\boldsymbol{X}, \boldsymbol{w}\rangle\left(p_{0}\right)=\left\langle\boldsymbol{X}_{x}, \boldsymbol{w}\right\rangle\left(p_{0}\right)=\left\langle\boldsymbol{X}_{y}, \boldsymbol{w}\right\rangle\left(p_{0}\right)=0 .
$$

The above condition is equivalent to the condition that $\boldsymbol{w} \in N_{p_{0}} M$ and $\boldsymbol{w} \in S_{t}^{1} \times S_{s}^{2}$. This means that $\boldsymbol{w}=\mu\left(\boldsymbol{e}_{1} \pm \boldsymbol{e}_{2}\right)=\overline{\boldsymbol{e}_{1} \pm \boldsymbol{e}_{2}}$.

By a Lorentzian motion on $A d S^{4}$, we may assume that $p_{0}=(0,1,0,0,0) \in A d S^{4}$. We can choose local coordinates such that $\boldsymbol{X}$ is given by the Monge form

$$
\boldsymbol{X}(x, y)=\left(f_{1}(x, y), f_{2}(x, y), f_{3}(x, y), x, y\right)
$$


with $f_{1}(0,0)=f_{3}(0,0)=0, f_{2}(0,0)=1$ and $f_{1_{x}}(0,0)=f_{1_{y}}(0,0)=f_{2_{x}}(0,0)=f_{2_{y}}(0,0)=$ $f_{3_{x}}(0,0)=f_{3_{y}}(0,0)=0$, so that we have $\boldsymbol{e}_{1}\left(p_{0}\right)=(1,0,0,0,0), \boldsymbol{e}_{2}\left(p_{0}\right)=(0,0,1,0,0)$. In this case we have

$$
\begin{array}{ccc}
f_{1_{x x}}(0,0)=-a\left(p_{0}\right), & f_{1_{x y}}(0,0)=-b\left(p_{0}\right), & f_{1_{y y}}(0,0)=-c\left(p_{0}\right), \\
f_{2_{x x}}(0,0)=e\left(p_{0}\right), & f_{2_{x y}}(0,0)=f\left(p_{0}\right), & f_{2_{y y}}(0,0)=g\left(p_{0}\right), \\
f_{3_{x x}}(0,0)=-1, & f_{3_{x y}}(0,0)=0, & f_{3_{y y}}(0,0)=-1 .
\end{array}
$$

Under the asumption (1) and taking into account that

$$
\operatorname{det} \mathcal{H}\left(h_{w}\right)(x, y)=\left|\begin{array}{cc}
\left\langle\boldsymbol{X}_{x x}, \boldsymbol{w}\right\rangle & \left\langle\boldsymbol{X}_{x y}, \boldsymbol{w}\right\rangle \\
\left\langle\boldsymbol{X}_{x y}, \boldsymbol{w}\right\rangle & \left\langle\boldsymbol{X}_{y y}, \boldsymbol{w}\right\rangle
\end{array}\right|=0
$$

and $\boldsymbol{w}\left(p_{0}\right)=(1,0, \pm 1,0,0)$, we have

$$
\begin{gathered}
\left|\begin{array}{cc}
\left\langle\left(f_{1_{x x}}, 0, f_{2_{x x}}, f_{3_{x x}}, 0\right), \boldsymbol{w}\left(p_{0}\right)\right\rangle & \left\langle\left(f_{1_{x y}}, 0, f_{2_{x y}}, f_{3_{x y}}, 0\right), \boldsymbol{w}\left(p_{0}\right)\right\rangle \\
\left\langle\left(f_{1_{x y}}, 0, f_{2_{x y}}, f_{3_{x y}}, 0\right), \boldsymbol{w}\left(p_{0}\right)\right\rangle & \left\langle\left(f_{1_{y y}}, 0, f_{2_{y y}}, f_{3_{y y}}, 0\right), \boldsymbol{w}\left(p_{0}\right)\right\rangle
\end{array}\right| \\
=\left|\begin{array}{cc}
\langle(-a, 0, e,-1,0),(1,0, \pm 1,0,0)\rangle & \langle(-b, 0, f, 0,0),(1,0, \pm 1,0,0)\rangle \\
\langle(-b, 0, f, 0,0),(1,0, \pm 1,0,0)\rangle & \langle(-c, 0, g,-1,0),(1,0, \pm 1,0,0)\rangle
\end{array}\right| \\
=\left|\begin{array}{ll}
a \pm e & b \pm f \\
b \pm f & c \pm g
\end{array}\right|=(a \pm e)(c \pm g)-(b \pm f)^{2}=0 .
\end{gathered}
$$

This is equivalent to the condition that $\mathcal{K}_{\ell}(1, \pm 1)\left(p_{0}\right)=0$ and $\boldsymbol{w}\left(p_{0}\right)=(1,0, \pm 1,0,0)$.

We now consider a point $p \in M$. As a corollary of the above proposition, we have the following theorem.

Theorem 3.2 Under the assumptions of Proposition 3.1, the following conditions are equivalent:

(1) There exists a $\boldsymbol{w} \in S_{t}^{1} \times S_{s}^{2}$ such that $p \in M$ is a degenerate singular point of nullcone height function $h_{w}$.

(2) The point $p \in M$ is a singular point of the $S_{t}^{1} \times S_{s}^{2}$-nullcone Legendrian Gauss map $\mathbb{N G}_{M}^{ \pm}$.

(3) $\mathcal{K}_{\ell}(1, \pm 1)(p)=0$.

Proof. By the general theory of unfoldings (cf., [18, 3]), (1) and (2) are equivalent. By the assertion (2) of Proposition 3.1, (1) and (3) are equivalent.

We say that a point $p_{0}=\left(x_{0}, y_{0}\right)$ is a lightlike parabolic point of $M$ if $\mathcal{K}_{\ell}(1,1)\left(p_{0}\right)=0$ or $\mathcal{K}_{\ell}(1,-1)\left(p_{0}\right)=0$. We study next the case when the $S_{t}^{1} \times S_{s}^{2}$-nullcone Legendrian Gauss map has the most degenerate singularities (i.e., it is constant).

Theorem 3.3 Let $M$ be a spacelike surface in $A d S^{4} \subset \mathbb{R}_{2}^{5}$.

(1) The $S_{t}^{1} \times S_{s}^{2}$-nullcone Legendrian Gauss map $\mathbb{N G}_{M}^{+}$(respectively, $\mathbb{N G}_{M}^{-}$) is constant if and only if there exists a unique null hyperplane $N H_{M}\left(\boldsymbol{v}^{+}, 0\right)$ (respectively, $\left.N H_{M}\left(\boldsymbol{v}^{-}, 0\right)\right)$ in $\mathbb{R}_{2}^{5}$, such that $M \subset N H\left(\boldsymbol{v}^{+}, 0\right) \cap A d S^{4}$ (respectively, $\left.M \subset N H\left(\boldsymbol{v}^{-}, 0\right) \cap A d S^{4}\right)$, where $\boldsymbol{v}^{ \pm}=\overline{\boldsymbol{e}_{1} \pm \boldsymbol{e}_{2}}(x, y)$ for any $(x, y) \in M$.

(2) Both of the $S_{t}^{1} \times S_{s}^{2}$-nullcone Legendrian Gauss maps $\mathbb{N} \mathbb{G}_{M}^{+}$and $\mathbb{N}_{M}^{-}$are constant if and only if $M$ is an open subset of

$$
N H\left(\overline{\boldsymbol{e}_{1}+\boldsymbol{e}_{2}}, 0\right) \cap N H_{M}\left(\overline{\boldsymbol{e}_{1}-\boldsymbol{e}_{2}}, 0\right) \cap A d S^{4} .
$$


Proof. (1) For convenience, we only consider the case when $\mathbb{N G}_{M}^{+}(x, y)=\overline{\boldsymbol{e}_{1}+\boldsymbol{e}_{2}}(x, y)$ is constant, so we have $d\left\langle\boldsymbol{X}, \overline{\boldsymbol{e}_{1}+\boldsymbol{e}_{2}}\right\rangle=\left\langle d \boldsymbol{X}, \overline{\boldsymbol{e}_{1}+\boldsymbol{e}_{2}}\right\rangle+\left\langle\boldsymbol{X}, d\left(\overline{\boldsymbol{e}_{1}+\boldsymbol{e}_{2}}\right)\right\rangle=0$. Therefore, $\left\langle\boldsymbol{X}, \overline{\boldsymbol{e}_{1}+\boldsymbol{e}_{2}}\right\rangle \equiv 0$ in $A d S^{4}$. This means that $M=\boldsymbol{X}(U) \subset N H_{M}\left(\boldsymbol{v}^{+}, 0\right)$, where $\boldsymbol{v}^{+}=$ $\overline{\boldsymbol{e}_{1}+\boldsymbol{e}_{2}}(x, y)$. For the converse assertion, suppose that there exists a null vector $\boldsymbol{v}$ and a real number $c$ such that $\boldsymbol{X}(U)=M \subset N H(\boldsymbol{v}, 0)$. Since $\langle\boldsymbol{X}(x, y), \boldsymbol{v}\rangle=0$, we have $d\langle\boldsymbol{X}(x, y), \boldsymbol{v}\rangle=0$. This means that $\boldsymbol{v}$ is a null normal vector of $M$. Thus we have $\overline{\boldsymbol{v}}=\overline{\boldsymbol{e}_{1} \pm \boldsymbol{e}_{2}}(x, y)$. This completes the proof of the assertion (1).

Since $\boldsymbol{v}^{+} \notin N H\left(\boldsymbol{v}^{-}, 0\right)$ and $\boldsymbol{v}^{-} \notin N H\left(\boldsymbol{v}^{+}, 0\right), N H\left(\boldsymbol{v}^{-}, 0\right)$ and $N H\left(\boldsymbol{v}^{+}, 0\right)$ intersect transversally. By the assertion (1), both of the $S_{t}^{1} \times S_{s}^{2}$-nullcone Legendrian Gauss maps $\mathbb{N} \mathbb{G}_{M}^{+}$and $\mathbb{N} G_{M}^{-}$are constant if and only if $M \subset N H\left(\boldsymbol{v}^{+}, 0\right) \cap N H\left(\boldsymbol{v}^{-}, 0\right)$. Here, the intersection is a spacelike affine 3 -space. Thus we have the assertion (2).

We analyze the intersection $N H\left(\boldsymbol{v}^{ \pm}, 0\right) \cap A d S^{4}$ when $\boldsymbol{v}^{ \pm}$is a null vector. Here we assume that both vectors $\boldsymbol{v}^{ \pm}$are null and such that $\left\langle\boldsymbol{v}^{+}, \boldsymbol{v}^{-}\right\rangle=-2$. By applying a semi-Euclidean motion on $\mathbb{R}_{2}^{5}$ if necessary, we may assume that $\boldsymbol{v}^{ \pm}=(1,0, \pm 1,0,0)$. For $\boldsymbol{v}^{+}=(1,0,1,0,0)$ the intersection is given by the equations $-x_{1}^{2}-x_{2}^{2}+x_{3}^{2}+x_{4}^{2}+x_{5}^{2}=-1,-x_{1}+x_{3}=0$, and we get

$$
N H\left(\boldsymbol{v}^{+}, 0\right) \cap A d S^{4}=\left\{\left(x_{1}, x_{2}, x_{3}, x_{4}, x_{5}\right) \in \mathbb{R}_{2}^{5} \mid-x_{2}^{2}+x_{4}^{2}+x_{5}^{2}=-1\right\} .
$$

This is $H^{2}(-1) \times \mathbb{R}$, where $H^{2}(-1)$ is a spacelike hyperboloid and the direction of the ruling $\mathbb{R}$ is $\boldsymbol{v}^{+}$. Therefore we call $N H\left(\boldsymbol{v}^{+}, 0\right) \cap A d S^{4}$ a lightlike hyperbolic cylinder. For $\boldsymbol{v}^{-}=(1,0,-1,0,0)$, we have a similar lightlike hyperbolic cylinder but with the lightlike direction $\boldsymbol{v}^{-}$. The intersection $N H\left(\boldsymbol{v}^{+}, 0\right) \cap N H\left(\boldsymbol{v}^{-}, 0\right) \cap A d S^{4}$ is a spacelike hyperboloid. For $\boldsymbol{v}^{ \pm}=(1,0, \pm 1,0,0)$, this is given by

$$
\left\{\left(0, x_{2}, 0, x_{3}, x_{4}, x_{5}\right) \in \mathbb{R}_{2}^{5} \mid-x_{2}^{2}+x_{4}^{2}+x_{5}^{2}=-1\right\} .
$$

\section{$4 \quad S_{t}^{1} \times S_{s}^{2}$-nullcone Legendrian Gauss maps}

In this section we consider the $S_{t}^{1} \times S_{s}^{2}$-nullcone Legendrian Gauss map of $M$ from the view point of Legendrian singularity theory. We give a brief review on Legendrian singularity theory mainly due to Arnol'd-Zakalyukin [1, 26]. Although the general theory has been described for any dimension, we shall just be concerned here with the 3-dimensional case. Since we only study local properties, we can consider the projective cotangent bundle $\pi: P T^{*}\left(\mathbb{R}^{3}\right) \longrightarrow \mathbb{R}^{3}$ with the canonical contact structure $K$. An immersion $i: L \rightarrow P T^{*}\left(\mathbb{R}^{3}\right)$ is said to be a Legendrian immersion if $\operatorname{dim} L=2$ and $d i_{q}\left(T_{q} L\right) \subset K_{i(q)}$ for any $q \in L$. We also call the map $\pi \circ i$ the Legendrian map and the set $W(i)=$ image $\pi \circ i$, the wave front of $i$. Moreover, $i$ (or, the image of $i$ ) is called the Legendrian lift of $W(i)$. The main tool of the theory of Legendrian singularities is the notion of generating families. Let $F:\left(\mathbb{R}^{k} \times \mathbb{R}^{3}, \mathbf{0}\right) \longrightarrow(\mathbb{R}, \mathbf{0})$ be a function germ. We say that $F$ is a Morse family of hypersurfaces if the mapping

$$
\Delta^{*} F=\left(F, \frac{\partial F}{\partial q_{1}}, \ldots, \frac{\partial F}{\partial q_{k}}\right):\left(\mathbb{R}^{k} \times \mathbb{R}^{3}, \mathbf{0}\right) \longrightarrow\left(\mathbb{R} \times \mathbb{R}^{k}, \mathbf{0}\right)
$$

is non-singular, where $(q, x)=\left(q_{1}, \ldots, q_{k}, x_{1}, x_{2}, x_{3}\right) \in\left(\mathbb{R}^{k} \times \mathbb{R}^{3}, \mathbf{0}\right)$. In this case we have a smooth 2-dimensional submanifold

$$
\Sigma_{*}(F)=\left\{(q, x) \in\left(\mathbb{R}^{k} \times \mathbb{R}^{3}, \mathbf{0}\right) \mid F(q, x)=\frac{\partial F}{\partial q_{1}}(q, x)=\cdots=\frac{\partial F}{\partial q_{k}}(q, x)=0\right\}
$$


and the map germ $\mathcal{L}_{F}:\left(\Sigma_{*}(F), \mathbf{0}\right) \longrightarrow P T^{*} \mathbb{R}^{3}$ defined by

$$
\mathcal{L}_{F}(q, x)=\left(x,\left[\frac{\partial F}{\partial x_{1}}(q, x): \frac{\partial F}{\partial x_{2}}(q, x): \frac{\partial F}{\partial x_{3}}(q, x)\right]\right)
$$

is a Legendrian immersion. Then we have the following fundamental theorem of Arnol'dZakalyukin $[1,26]$.

Proposition 4.1 All Legendrian submanifold germs in $P T^{*} \mathbb{R}^{4}$ are constructed by the above method.

We call $F$ a generating family of $\mathcal{L}_{F}$. Therefore the corresponding wave front is

$$
W\left(\mathcal{L}_{F}\right)=\left\{x \in \mathbb{R}^{4} \mid \exists q \in \mathbb{R}^{k} \text { such that } F(q, x)=\frac{\partial F}{\partial q_{1}}(q, x)=\cdots=\frac{\partial F}{\partial q_{k}}(q, x)=0\right\} .
$$

We write $\mathcal{D}_{F}=W\left(\mathcal{L}_{F}\right)$ and we call it the discriminant set of $F$. By Proposition 3.1, the image of $S_{t}^{1} \times S_{s}^{2}$-nullcone Legendrian Gauss map $\mathbb{N G}_{M}^{ \pm}(U)$ is the discriminant set of the nullcone height function $H$. We have the following proposition.

Proposition 4.2 The nullcone height function $H$ is a Morse family of hypersurfaces.

Proof. We consider the nullcone height function $H: M \times S_{t}^{1} \times S_{s}^{2} \longrightarrow \mathbb{R}$. For any $\boldsymbol{w}=$ $\left(\cos \theta, \sin \theta, w_{3}, w_{4}, w_{5}\right) \in S_{t}^{1} \times S_{s}^{2}$, we assume that $w_{3}>0$, so we can write

$$
H(p, \boldsymbol{w})=-x_{1}(p) \cos \theta-x_{2}(p) \sin \theta+x_{3}(p) \sqrt{1-w_{4}^{2}-w_{5}^{2}}+x_{4}(p) w_{4}+x_{5}(p) w_{5},
$$

where $\boldsymbol{X}(p)=\left(x_{1}(p), x_{2}(p), x_{3}(p), x_{4}(p), x_{5}(p)\right)$. We now prove that the mapping

$$
\Delta^{*} H=\left(H, \frac{\partial H}{\partial x}, \frac{\partial H}{\partial y}\right): U \times S_{t}^{1} \times S_{s}^{2} \longrightarrow \mathbb{R}^{3}
$$

is non-singular at any point in $\left(\Delta^{*}\right)^{-1}(0)=\Sigma_{*}(H)$. The Jacobian matrix of $\Delta^{*} H$ is given by

$$
\left(\begin{array}{ccccc}
\left\langle\boldsymbol{X}_{x}, \boldsymbol{w}\right\rangle & \left\langle\boldsymbol{X}_{y}, \boldsymbol{w}\right\rangle & x_{1} \sin \theta-x_{2} \cos \theta & -x_{3} \frac{w_{4}}{w_{3}}+x_{4} & -x_{3} \frac{w_{5}}{w_{3}}+x_{5} \\
\left\langle\boldsymbol{X}_{x x}, \boldsymbol{w}\right\rangle & \left\langle\boldsymbol{X}_{x y}, \boldsymbol{w}\right\rangle & x_{1, x} \sin \theta-x_{2, x} \cos \theta & -x_{3, x} \frac{w_{4}}{w_{3}}+x_{4, x} & -x_{3, x} \frac{w_{5}}{w_{3}}+x_{5, x} \\
\left\langle\boldsymbol{X}_{x y}, \boldsymbol{w}\right\rangle & \left\langle\boldsymbol{X}_{y y}, \boldsymbol{w}\right\rangle & x_{1, y} \sin \theta-x_{2, y} \cos \theta & -x_{3, y} \frac{w_{4}}{w_{3}}+x_{4, y} & -x_{3, y} \frac{w_{5}}{w_{3}}+x_{5, y}
\end{array}\right) .
$$

By a straight forward calculation, the determinant of the matrix

$$
A=\left(\begin{array}{cc}
-x_{3, x} \frac{w_{4}}{w_{3}}+x_{4, x} & -x_{3, x} \frac{w_{5}}{w_{3}}+x_{5, x} \\
-x_{3, y} \frac{w_{4}}{w_{3}}+x_{4, y} & -x_{3, y} \frac{w_{5}}{w_{3}}+x_{5, y}
\end{array}\right) .
$$

can be seen to be equal to

$$
\frac{w_{5}}{w_{3}}\left(x_{3, x} x_{4, y}-x_{4, x} x_{3, y}\right)+\frac{w_{4}}{w_{3}}\left(x_{5, x} x_{3, y}-x_{3, x} x_{5, y}\right)+\left(x_{4, x} x_{5, y}-x_{5, x} x_{4, x}\right) .
$$

Since $\boldsymbol{X}(U)=M$ is a spacelike surface, the surface in Euclidean space $\{(0,0)\} \times \mathbb{R}^{3}$ parameterized by $\left(0,0, x_{3}(x, y), x_{4}(x, y), x_{5}(x, y)\right)$ is a regular surface. It follows that the above 
determinant vanishes if and only if the vector $\left(0, w_{3}, w_{4}, w_{5}\right)$ is tangent to the surface $M_{0}=$ $\left\{\left(0,0, x_{3}(x, y), x_{4}(x, y), x_{5}(x, y)\right) \mid(x, y) \in U\right\}$ in $\{(0,0)\} \times \mathbb{R}^{3}$. We consider the canonical projection $\pi_{3}: \mathbb{R}_{2}^{5} \longrightarrow\{(0,0)\} \times \mathbb{R}^{3}$. Since $M=\boldsymbol{X}(U)$ is a spacelike surface, $\pi_{3} \mid M$ is a diffeomorphism onto $M_{0}$. The vector $\boldsymbol{w}=\left(\cos \theta, \sin \theta, w_{1}, w_{2}, w_{3}\right)$ is a null normal vector of $M$ and does not belong to $\operatorname{Ker} d \pi_{3} \mid M$, therefore $d \pi_{3}(\boldsymbol{w})=\left(0,0, w_{1}, w_{2}, w_{3}\right)$ is not tangent to $M_{0}$.

It follows from Proposition 4.2 that the images of the $S_{t}^{1} \times S_{s}^{2}$-nullcone Legendrian Gauss maps $\mathbb{N}_{M}^{ \pm}(U)$ are wave fronts and the nullcone height function $H$ is a generating families of the Legendrian lifts of $\mathbb{N}_{M}^{ \pm}(U)$, at least locally. By the assertion (1) of Proposition 3.1, we have

$$
\Sigma_{*}(H)=\left\{(p, \boldsymbol{w}) \in M \times S_{t}^{1} \times S_{s}^{2} \mid \boldsymbol{w}=\overline{\boldsymbol{e}_{1} \pm \boldsymbol{e}_{2}}(p)\right\} .
$$

We now consider the coordinate neighborhood

$$
I \times U_{3}^{+}=\left\{\left(\cos \bar{\theta}, \sin \bar{\theta}, w_{3}, w_{4}, w_{5}\right) \in S_{t}^{1} \times S_{s}^{2} \mid \bar{\theta} \in I=(0,2 \pi), w_{3}>0\right\}
$$

of $S_{t}^{1} \times S_{s}^{2}$. By the construction of the Legendrian immersion in Proposition 4.1, we have the Legendrian immersion $\mathcal{L}_{H}: V \longrightarrow P T^{*}\left(S_{t}^{1} \times S_{s}^{2}\right) \mid I \times U_{3}^{+}$defined by

$$
\mathcal{L}_{H}(p)=\left(\overline{\boldsymbol{e}_{1} \pm \boldsymbol{e}_{2}}(p),\left[\lambda(p) \sin (\theta(p)-\bar{\theta}(p)):-\frac{w_{4}(p)}{w_{3}(p)} x_{3}(p)+x_{4}(p):-\frac{w_{5}(p)}{w_{3}(p)} x_{3}(p)+x_{5}(p)\right]\right)
$$

where $V=\left(\mathbb{N} \mathbb{G}_{M}^{ \pm}\right)^{-1}\left(I \times U_{3}^{+}\right)$and we write

$$
\overline{\boldsymbol{e}_{1} \pm \boldsymbol{e}_{2}}(p)=\left(\cos \bar{\theta}(p), \sin \bar{\theta}(p), w_{3}(p), w_{4}(p), w_{5}(p)\right)
$$

and

$$
\boldsymbol{X}(p)=\left(\lambda(p) \cos \theta(p), \lambda(p) \sin \theta(p), x_{3}(p), x_{4}(p), x_{5}(p)\right) .
$$

In the other coordinate neighborhoods, we have a similar expression for the Legendrian lift. This expression will be used in the next section.

\section{$5 \quad S_{+}^{2}$-nullcone Lagrangian Gauss maps}

In this section we describe some of the geometric properties of the $S_{+}^{2}$-nullcone Lagrangian Gauss map $\widehat{\mathbb{N G}}_{M}^{ \pm}$of a spacelike surface in $M=\boldsymbol{X}(U)$. We fix the coordinate neighborhood, $I \times U_{3}^{+}$, as in the last paragraph of the previous section. By the local triviality of the projective cotangent bundle, we have $P T^{*}\left(S_{t}^{1} \times S_{s}^{2}\right) \mid I \times U_{3}^{+} \equiv\left(I \times U_{3}^{+}\right) \times \mathbb{R} P^{*}\left(\mathbb{R} \times \mathbb{R}^{2}\right)$. We assume that $\xi_{3} \neq 0$ for $(\boldsymbol{w},[\xi])=\left(\left(\bar{\theta}, \sqrt{1-w_{4}^{2}-w_{5}^{2}}, w_{4}, w_{5}\right),\left[\xi_{3}: \xi_{4}: \xi_{5}\right]\right) \in\left(I \times U_{3}^{+}\right) \times \mathbb{R} P^{*}\left(\mathbb{R} \times \mathbb{R}^{2}\right)$. Under this assumption, we have $\left(\boldsymbol{w},\left[\xi_{3}: \xi_{4}: \xi_{5}\right]\right)=\left(\boldsymbol{w},\left[1: \frac{\xi_{4}}{\xi_{3}}: \frac{\xi_{5}}{\xi_{3}}\right]\right)$. Therefore the canonical contact form on $P T^{*}\left(S_{t}^{1} \times S_{s}^{2}\right) \mid I \times U_{3}^{+}$is given by $\alpha=d \bar{\theta}-\sum_{i=4}^{5} \eta_{i} d w_{i}$, where $\eta_{i}=\frac{\xi_{i}}{\xi_{3}}$. It follows that there exists a contact morphism $\Phi: P T^{*}\left(S_{t}^{1} \times S_{s}^{2}\right)\left|\left(I \times U_{3}^{+}\right) \cap\left\{\xi_{3} \neq 0\right\} \longrightarrow I \times T^{*} S_{+}^{2}\right|\left\{w_{3}>0\right\}$ defined by

$$
\Phi\left(\left(\theta, w_{3}, w_{4}, w_{5}\right),\left[\xi_{3}: \xi_{4}: \xi_{5}\right]\right)=\left(\theta,\left(0,1, w_{3}, w_{4}, w_{5}\right),\left(\frac{\xi_{4}}{\xi_{3}}, \frac{\xi_{5}}{\xi_{3}}\right)\right)
$$

where the contact structure on $I \times T^{*} S_{+}^{2} \mid\left\{w_{3}>0\right\}$ is also given by $\alpha$. We now consider the symplectic manifold $T^{*} S_{+}^{2} \mid\left\{w_{3}>0\right\}$ with the canonical symplectic structure $\omega=\sum_{i=4}^{5} d \eta_{i} \wedge d w_{i}$. 
On the other hand, we have $\Sigma_{*}(H)=\left\{\left(p, \overline{\boldsymbol{e}_{1} \pm \boldsymbol{e}_{2}}(p)\right) \mid p \in M\right\}$ by Propsition 3.1. By the definition of $\Sigma_{*}(H)$, we have

$$
0=H\left(p, \overline{\boldsymbol{e}_{1} \pm \boldsymbol{e}_{2}}(p)\right)=\left\langle\boldsymbol{X}(p), \overline{\boldsymbol{e}_{1} \pm \boldsymbol{e}_{2}}(p)\right\rangle=-\lambda(p) \cos (\theta(p)-\bar{\theta}(p))+\left(\boldsymbol{X}_{E}(p), \boldsymbol{w}_{E}(p)\right),
$$

where $\boldsymbol{X}_{E}(p)=\left(x_{3}(p), x_{4}(p), x_{5}(p)\right), \boldsymbol{w}_{E}(p)=\left(w_{3}(p), w_{4}(p), w_{5}(p)\right)$ and $(\cdot, \cdot)$ is the canonical Euclidean scalar product of $\mathbb{R}^{3}$. Therefore, we have

$$
\cos (\theta(p)-\bar{\theta}(p)) \leq \frac{\sqrt{\lambda^{2}(p)-1}}{\lambda(p)}<1,
$$

and thus $\lambda(p) \sin (\theta(p)-\bar{\theta}(p)) \neq 0$, so that $\mathcal{L}_{H}(V) \subset P T^{*}\left(S_{t}^{1} \times S_{s}^{2}\right) \mid\left(I \times U_{3}^{+}\right) \cap\left\{\xi_{3} \neq 0\right\}$. Let $\Pi: I \times T^{*} S_{+}^{2} \longrightarrow T^{*} S_{+}^{2}$ be the canonical projection. We define a mapping $L_{H}: V \longrightarrow T^{*} S_{+}^{2}$ by $L_{H}(p)=\Pi \circ \Phi \circ \mathcal{L}_{H}(p)$. Since $\Phi$ is a contact morphism, $\Phi \circ \mathcal{L}_{H}$ is a Legendrian immersion. It follows that $L_{H}$ is a Lagrangian immersion. Moreover, we have

$$
L_{H}(p)=\left(\widehat{\tau} \circ \overline{\boldsymbol{e}_{1} \pm \boldsymbol{e}_{2}}(p),\left(\frac{\xi_{4}}{\xi_{3}}, \frac{\xi_{5}}{\xi_{3}}\right)\right)=\left(\widehat{\mathbb{N G}}_{M}^{ \pm}(p),\left(\frac{\xi_{4}}{\xi_{3}}, \frac{\xi_{5}}{\xi_{3}}\right)\right) .
$$

This means that $\widehat{\mathbb{N G}}_{M}^{ \pm}$is a Lagrangian map. Since $L_{H}=\Pi \circ \Phi \circ \mathcal{L}_{H}$, it is easy to show that $p \in M$ is a singular point of $\widehat{\mathbb{N G}}_{M}^{ \pm}$if and only if it is a singular point of $\mathbb{N G}_{M}^{ \pm}$. This fact is a well-known and simple fact on the relation between Lagrangian singularities and Legendrian singularities in general. Therefore we have the following proposition.

Proposition 5.1 For a point $p \in M$, the following conditions are equivalent:

(1) $\mathcal{K}_{\ell}(1, \pm 1)(p)=0$.

(2) $\overline{\mathcal{K}}_{\ell}(1, \pm 1)(p)=0$.

(3) The point $p \in M$ is a singular point of $\mathbb{N G}_{M}^{ \pm}$.

(4) The point $p \in M$ is a singular point of $\widehat{\mathbb{N G}}_{M}^{ \pm}$.

(5) $\widehat{\mathcal{K}}_{\ell}^{ \pm}(p)=0$.

\section{Contact with lightlike hyperbolic cylinders}

We analyze in this section the geometrical meaning of the singularities of the $S_{t}^{1} \times S_{s}^{2}$-nullcone Legendrian Gauss map on a spacelike surface $\boldsymbol{X}(U)=M$ in Anti de Sitter 4-space. For this purpose we shall study the contacts between spacelike surfaces and lightlike hyperbolic cylinders as in the classical differential geometry. In the first place, we briefly review the theory of contact due to Montaldi [21]. Let $X_{i}, Y_{i}(i=1,2)$ be submanifolds of $\mathbb{R}^{n}$ with $\operatorname{dim} X_{1}=\operatorname{dim} X_{2}$ and $\operatorname{dim} Y_{1}=\operatorname{dim} Y_{2}$. We say that the contact of $X_{1}$ and $Y_{1}$ at $y_{1}$ is of the same type than the contact of $X_{2}$ and $Y_{2}$ at $y_{2}$ if there is a diffeomorphism germ $\Phi:\left(\mathbb{R}^{n}, y_{1}\right) \longrightarrow\left(\mathbb{R}^{n}, y_{2}\right)$ such that $\Phi\left(X_{1}\right)=X_{2}$ and $\Phi\left(Y_{1}\right)=Y_{2}$. In this case we write $K\left(X_{1}, Y_{1} ; y_{1}\right)=K\left(X_{2}, Y_{2} ; y_{2}\right)$. It is clear that in this definition $\mathbb{R}^{n}$ could be replaced by any manifold. In [21], Montaldi gives a characterization of the notion of contact by using the terminology of singularity theory.

Theorem 6.1 Let $X_{i}, Y_{i}(i=1,2)$ be submanifolds of $\mathbb{R}^{n}$ with $\operatorname{dim} X_{1}=\operatorname{dim} X_{2}$ and $\operatorname{dim} Y_{1}=$ $\operatorname{dim} Y_{2}$. Let $g_{i}:\left(X_{i}, x_{i}\right) \longrightarrow\left(\mathbb{R}^{n}, y_{i}\right)$ be immersion germs and $f_{i}:\left(\mathbb{R}^{n}, y_{i}\right) \longrightarrow\left(\mathbb{R}^{p}, 0\right)$ be submersion germs with $\left(Y_{i}, y_{i}\right)=\left(f_{i}^{-1}(0), y_{i}\right)$. Then $K\left(X_{1}, Y_{1} ; y_{1}\right)=K\left(X_{2}, Y_{2} ; y_{2}\right)$ if and only if $f_{1} \circ g_{1}$ and $f_{2} \circ g_{2}$ are $\mathcal{K}$-equivalent. For the definition of the $\mathcal{K}$-equivalence among smooth map-germs, see [18, 19]. 
Consider the function $\mathcal{H}: A d S^{4} \times\left(S_{t}^{1} \times S_{s}^{2}\right) \longrightarrow \mathbb{R}$ defined by $\mathcal{H}(\boldsymbol{x}, \boldsymbol{v})=\langle\boldsymbol{x}, \boldsymbol{v}\rangle$, where $\left(v_{1}, v_{2}, v_{3}, v_{4}, v_{5}\right)$. For any $\boldsymbol{v}_{0}=\left(v_{0,1}, v_{0,2}, v_{0,3}, v_{0,4}, v_{0,5}\right) \in S_{t}^{1} \times S_{s}^{2}$, we denote that $\mathfrak{h}_{v_{0}}(\boldsymbol{x})=$ $\mathcal{H}\left(\boldsymbol{x}, \boldsymbol{v}_{0}\right)$. Then the subset $\mathfrak{h}_{v_{0}}^{-1}(0)=N H\left(\boldsymbol{v}_{0}, 0\right) \cap A d S^{4}$ is lightlike hyperbolic cylinder. Given $p_{0}=\boldsymbol{X}\left(x_{0}, y_{0}\right) \in U$, we can take the lightlike vector $\boldsymbol{v}_{0}^{ \pm}=\overline{\boldsymbol{e}_{1} \pm \boldsymbol{e}_{2}}\left(p_{0}\right)$, then we have

$$
\mathfrak{h}_{v_{0}^{ \pm}} \circ \boldsymbol{X}\left(p_{0}\right)=\mathcal{H} \circ\left(\boldsymbol{X} \times i d_{L C_{0}^{*}}\right)\left(\left(p_{0}\right), \boldsymbol{v}_{0}^{ \pm}\right)=H\left(\left(x_{0}, y_{0}\right), \boldsymbol{v}_{0}^{ \pm}\right)=0 .
$$

We also have the relations

$$
\frac{\partial \mathfrak{h}_{v_{0}^{ \pm}} \circ \boldsymbol{X}}{\partial x}\left(p_{0}\right)=\frac{\partial H}{\partial x}\left(\left(p_{0}\right), \boldsymbol{v}_{0}^{ \pm}\right)=0
$$

and

$$
\frac{\partial \mathfrak{h}_{v_{0}^{ \pm}} \circ \boldsymbol{X}}{\partial y}\left(p_{0}\right)=\frac{\partial H}{\partial y}\left(\left(p_{0}\right), \boldsymbol{v}_{0}^{ \pm}\right)=0 .
$$

This means that the lightlike hyperbolic cylinder $\mathfrak{h}_{v_{0}^{ \pm}}^{-1}(0)=N H\left(\boldsymbol{v}_{0}^{ \pm}, 0\right) \cap A d S^{4}$ is tangent to $M=\boldsymbol{X}(U)$ at $p_{0}=\boldsymbol{X}\left(x_{0}, y_{0}\right)$ in the Anti de Sitter 4-space $A d S^{4}$. In this case, we call each $N H\left(\boldsymbol{v}_{0}^{ \pm}, 0\right) \cap A d S^{4}$ a tangent lightlike hyperbolic cylinder of $M=\boldsymbol{X}(U)$ at $p_{0}=\boldsymbol{X}\left(x_{0}, y_{0}\right)$ in $A d S^{4}$. We denote it as $T H C\left(\boldsymbol{v}^{ \pm}, M, p_{0}\right)$. Let $\boldsymbol{v}_{1}, \boldsymbol{v}_{2}$ be two null vectors. The intersection $N H\left(\boldsymbol{v}_{0}^{+}, 0\right) \cap N H\left(\boldsymbol{v}_{0}^{-},\right) \cap A d S^{4}$ is a spacelike hyperboloid tangent to $M=\boldsymbol{X}(U)$ at $p_{0}=$ $\boldsymbol{X}\left(x_{0}, y_{0}\right)$. We call it the tangent spacelike hyperboloid of $M=\boldsymbol{X}(U)$ at $p_{0}=\boldsymbol{X}\left(x_{0}, y_{0}\right)$. We write it as $T S H\left(\boldsymbol{v}^{ \pm}, M, p_{0}\right)$. If $\boldsymbol{v}_{1}, \boldsymbol{v}_{2}$ are linearly dependent, then the corresponding null hyperplanes $N H\left(\boldsymbol{v}_{1}, 0\right)$ and $N H\left(\boldsymbol{v}_{2}, 0\right)$ are coincident. Then we have the following lemma, whose proof is traightforward.

Lemma 6.2 Let $\boldsymbol{X}: U \longrightarrow A d S^{4}$ be an immersion such that $M=\boldsymbol{X}(U)$ is a spacelike surface and $\sigma= \pm$. Consider two points $p_{1}=\boldsymbol{X}\left(x_{1}, y_{1}\right), p_{2}=\boldsymbol{X}\left(x_{2}, y_{2}\right)$. Then $\mathbb{N}_{G}^{\sigma}\left(p_{1}\right)=\mathbb{N} \mathbb{G}_{M}^{\sigma}\left(p_{2}\right)$ if and only if $T H C\left(\boldsymbol{v}_{1}^{\sigma}, M, p_{1}\right)=T H C\left(\boldsymbol{v}_{2}^{\sigma}, M, p_{2}\right)$. Here, $v_{i}^{ \pm}=\overline{\boldsymbol{e}_{1} \pm \boldsymbol{e}_{2}}\left(p_{i}\right)$ for $i=1,2$.

We denote $\mathcal{E}_{n}$ the local ring of function germs $\left(\mathbb{R}^{n}, \mathbf{0}\right) \longrightarrow \mathbb{R}$ with the unique maximal ideal $\mathfrak{M}_{n}=\left\{h \in \mathcal{E}_{n} \mid h(0)=0\right\}$. Let $F, G:\left(\mathbb{R}^{k} \times \mathbb{R}^{n}, \mathbf{0}\right) \longrightarrow(\mathbb{R}, \mathbf{0})$ be function germs. We say that $F$ and $G$ are $P$-K $\mathcal{K}$-equivalent if there exists a diffeomorphism germ $\Psi:\left(\mathbb{R}^{k} \times \mathbb{R}^{n}, \mathbf{0}\right) \longrightarrow\left(\mathbb{R}^{k} \times \mathbb{R}^{n}, \mathbf{0}\right)$ of the form $\Psi(x, u)=\left(\psi_{1}(q, x), \psi_{2}(x)\right)$ for $(q, x) \in\left(\mathbb{R}^{k} \times \mathbb{R}^{n}, \mathbf{0}\right)$ such that $\Psi^{*}\left(\langle F\rangle_{\mathcal{E}_{k+n}}\right)=\langle G\rangle_{\mathcal{E}_{k+n}}$. Here $\Psi^{*}: \mathcal{E}_{k+n} \longrightarrow \mathcal{E}_{k+n}$ is the pull back $\mathbb{R}$-algebra isomorphism defined by $\Psi^{*}(h)=h \circ \Psi$.

We now apply the usual tools for the study of the contacts between spacelike surfaces and lightlike hyperbolic cylinders. Let $\mathbb{N}_{M, i}^{\sigma}:\left(U,\left(x_{i}, y_{i}\right)\right) \longrightarrow\left(S_{t}^{1} \times S_{s}^{2}, \boldsymbol{v}_{i}^{\sigma}\right)(i=1,2)$ be two $\left(S_{t}^{1} \times S_{s}^{2}\right)$-nullcone Legendrian Gauss map germs of spacelike surface germs $\boldsymbol{X}_{i}:\left(U,\left(x_{i}, y_{i}\right)\right) \longrightarrow$ $\left(A d S^{4}, p_{i}\right)$, where $\sigma= \pm$. We say that $\mathbb{N} \mathbb{G}_{M, 1}^{\sigma}$ and $\mathbb{N G}_{M, 2}^{\sigma}$ are $\mathcal{A}$-equivalent if there exist diffeomorphism germs $\left.\phi:\left(U,\left(x_{1}, y_{1}\right)\right) \longrightarrow\left(U, x_{2}, y_{2}\right)\right)$ and $\Phi:\left(S_{t}^{1} \times S_{s}^{2}, \boldsymbol{v}_{1}^{\sigma}\right) \longrightarrow\left(S_{t}^{1} \times S_{s}^{2}, \boldsymbol{v}_{2}^{\sigma}\right)$ such that $\Phi \circ \mathbb{N G}_{M, 1}^{\sigma}=\mathbb{N G}_{M, 2}^{\sigma} \circ \phi$. Let $H_{i}:\left(U \times S_{t}^{1} \times S_{s}^{2},\left(\left(x_{i}, y_{i}\right), \boldsymbol{v}_{i}^{\sigma}\right)\right) \longrightarrow \mathbb{R}$ be the nullcone height function germ of $\boldsymbol{X}_{i}$. We denote $h_{i, v_{i}^{\sigma}}(u)=H_{i}\left(u, \boldsymbol{v}_{i}^{\sigma}\right)$, then we have $h_{i, v_{i}^{ \pm}}(u)=\mathfrak{h}_{v_{i}^{ \pm}} \circ \boldsymbol{X}_{i}(u)$. By Theorem 5.1, $K\left(\boldsymbol{X}_{1}(U), N H\left(\boldsymbol{v}^{\sigma},-1\right), \boldsymbol{v}_{1}^{\sigma}\right)=K\left(\boldsymbol{X}_{2}(U), N H\left(\boldsymbol{v}^{\sigma},-1\right), \boldsymbol{v}_{2}^{\sigma}\right)$ if and only if $h_{1, v_{1}}$ and $h_{1, v_{2}}$ are $\mathcal{K}$-equivalent. We define the local ring by

$$
Q^{ \pm}\left(\boldsymbol{X},\left(x_{0}, y_{0}\right)\right)=\frac{C_{\left(x_{0}, y_{0}\right)}^{\infty}(U)}{\left\langle\left\langle\boldsymbol{X}(x, y), \overline{\boldsymbol{e}_{1} \pm \boldsymbol{e}_{2}}\left(x_{0}, y_{0}\right)\right\rangle\right\rangle_{C_{\left(x_{0}, y_{0}\right)}^{\infty}(U)}},
$$

where $C_{\left(x_{0}, y_{0}\right)}^{\infty}(U)$ is the local ring of function germs at $\left(x_{0}, y_{0}\right)$ with the unique maximal ideal $\mathfrak{M}_{\left(x_{0}, y_{0}\right)}(U)$. By similar arguments to those of the proof for Theorem 6.3 in [9], we can show the following theorem: 
Theorem 6.3 Let $\boldsymbol{X}_{i}:\left(U,\left(x_{i}, y_{i}\right)\right) \longrightarrow\left(A d S^{4}, \boldsymbol{X}_{i}\left(\left(x_{i}, y_{i}\right)\right)\right)(i=1,2)$ be spacelike surface germs such that the corresponding Legendrian lift germs of the $S_{t}^{1} \times S_{s}^{2}$-nullcone Legendrian Gauss map germs are Legendrian stable and $\sigma= \pm$. Then the following conditions are equivalent:

(1) $S_{t}^{1} \times S_{s}^{2}$-nullcone Legendrian Gauss map germs $\mathbb{N G}_{1}^{\sigma}$ and $\mathbb{N}_{\mathbb{G}_{2}^{\sigma}}^{\sigma}$ are $\mathcal{A}$-equivalent.

(2) $H_{1}$ and $H_{2}$ are $P$-K-equivalent.

(3) $h_{1, v_{1}}$ and $h_{1, v_{2}}$ are $\mathcal{K}$-equivalent.

(4) $K\left(\boldsymbol{X}_{1}(U), T H C\left(\boldsymbol{v}^{\sigma}, \boldsymbol{X}_{1}\left(U_{1}\right)\right), \boldsymbol{X}\left(x_{1}, y_{1}\right)\right)=K\left(\boldsymbol{X}_{2}(U), T H C\left(\boldsymbol{v}^{\sigma}, \boldsymbol{X}_{2}(U), \boldsymbol{X}_{2}\left(x_{2}, y_{2}\right)\right)\right)$.

(5) $Q^{\sigma}\left(\boldsymbol{X}_{1},\left(x_{1}, y_{1}\right)\right)$ and $Q^{\sigma}\left(\boldsymbol{X}_{2},\left(x_{2}, y_{2}\right)\right)$ are isomorphic as $\mathbb{R}$-algebras.

For a spacelike surface germ $\boldsymbol{X}:\left(U,\left(x_{0}, y_{0}\right)\right) \longrightarrow\left(A d S^{4}, \boldsymbol{X}\left(x_{0}, y_{0}\right)\right)$, we call each set

$$
\left(\boldsymbol{X}^{-1}\left(T H C\left(\boldsymbol{v}^{\sigma}, \boldsymbol{X}(U), \boldsymbol{X}\left(x_{0}, y_{0}\right)\right)\right),\left(x_{0}, y_{0}\right)\right)
$$

a tangent lightlike hyperpbolic cylinder indicatrix germ of $\boldsymbol{X}$, where $\boldsymbol{v}^{ \pm}=\overline{\boldsymbol{e}_{1} \pm \boldsymbol{e}_{2}}\left(x_{0}, y_{0}\right)$. Moreover, by the above results, we adopt some basic invariants from the singularity theory on function germs. We need $\mathcal{K}$-invariants for function germ. The local ring of a function germ is a complete $\mathcal{K}$-invariant for generic function germs. It is, however, not a numerical invariant. The $\mathcal{K}$-codimension (or, Tyurina number) of a function germ is a numerical $\mathcal{K}$-invariant of function germs [18]. We denote it by

$$
\operatorname{L-ord}^{ \pm}\left(\boldsymbol{X},\left(x_{0}, y_{0}\right)\right)=\operatorname{dim}_{\mathbb{R}} \frac{C_{\left(x_{0}, y_{0}\right)}^{\infty}(U)}{\left\langle h_{v_{0}^{ \pm}}(x, y), h_{v_{0}^{ \pm}, x}(x, y), h_{v_{0}^{ \pm}, y}(x, y)\right\rangle} .
$$

Usually L-ord ${ }^{\sigma}\left(\boldsymbol{X}, u_{0}\right)$ is called the $\mathcal{K}$-codimension of $h_{v_{0}^{\sigma}}$, where $\sigma= \pm$. However, we call it the order of contact with the tangent lightlike hyperbolic cylinder at $\boldsymbol{X}\left(x_{0}, y_{0}\right)$. We also have the notion of corank of function germs.

$$
\operatorname{L-corank}^{\sigma}\left(\boldsymbol{X},\left(x_{0}, y_{0}\right)\right)=2-\operatorname{rank} \operatorname{Hess}\left(h_{v_{0}^{\sigma}}\left(x_{0}, y_{0}\right)\right),
$$

where $\boldsymbol{v}_{0}^{ \pm}=\overline{\boldsymbol{e}_{1} \pm \boldsymbol{e}_{2}}\left(x_{0}, y_{0}\right)$. By Proposition 2.1, $\boldsymbol{X}\left(x_{0}, y_{0}\right)$ is a $L^{\sigma}$-parabolic point if and only if $\mathrm{L}_{\text {-corank }}{ }^{\sigma}\left(\boldsymbol{X},\left(x_{0}, y_{0}\right)\right) \geq 1$. Moreover $\boldsymbol{X}\left(x_{0}, y_{0}\right)$ is a lightlike umbilic point if and only if $\operatorname{L-corank}^{\sigma}\left(\boldsymbol{X},\left(x_{0}, y_{0}\right)\right)=2$.

On the other hand, a function germ $f:\left(\mathbb{R}^{n-1}, \boldsymbol{a}\right) \longrightarrow \mathbb{R}$ has the $A_{k}$-type singularity if $f$ is $\mathcal{K}$-equivalent to the germ $\pm u_{1}^{2} \pm \cdots \pm u_{n-2}^{2}+u_{n-1}^{k+1}$. If $\operatorname{L-corank}^{\sigma}\left(\boldsymbol{X},\left(x_{0}, y_{0}\right)\right)=1$, the nullcone height function $h_{v_{0}^{\sigma}}$ has the $A_{k^{-}}$-type singularity at $\left(x_{0}, y_{0}\right)$ in generic. In this case we have $\mathrm{H}-\operatorname{ord}^{\sigma}\left(\boldsymbol{X}, u_{0}\right)=k$. This number $k$ is equal to the order of contact in the classical sense (cf., $[4])$. This is the reason why we call ${\mathrm{L}-\operatorname{ord}^{\sigma}}^{\sigma}\left(\boldsymbol{X},\left(x_{0}, y_{0}\right)\right)$ the order of contact with the tangent lightlike hyperbolic cylinder at $\boldsymbol{X}\left(x_{0}, y_{0}\right)$.

\section{$7 \quad$ Singularities of $S_{t}^{1} \times S_{s}^{2}$-nullcone Legendrian Gauss maps}

In this section we study the generic singularities of $S_{t}^{1} \times S_{s}^{2}$-nullcone Legendrian Gauss maps. We consider the space of spacelike embeddings $\operatorname{Emb}_{s}\left(U, A d S^{4}\right)$ with the Whitney $C^{\infty}$-topology, where $U \subset \mathbb{R}^{2}$ is an open subset. By standard arguments analogous to those used in [9, 13], we obtain the following theorem as a corollary of Lemma 6 in Wassermann [24]. (See also Montaldi $[22])$. 
Theorem 7.1 There exists an open dense subset $\mathcal{O} \subset \operatorname{Emb}_{s}\left(U, A d S^{4}\right)$ such that for any $\boldsymbol{X} \in$ $\mathcal{O}$, the following conditions hold:

(1) Each lightlike parabolic set $\mathcal{K}_{\ell}(1, \sigma 1)^{-1}(0)$ is a regular curve. We call such a curve the lightlike parabolic curve.

(2) The image of the $S_{t}^{1} \times S_{s}^{2}$-nullcone Legendrian Gauss map $\mathbb{N}_{M}^{\sigma}(U)$ along the lightlike parabolic curve is a cuspidaledge except at isolated points. At these points $\mathbb{N}_{M}^{\sigma}(U)$ is a swallowtail.

Here, $\sigma= \pm$ and a map germ $f:\left(\mathbb{R}^{2}, \boldsymbol{a}\right) \longrightarrow\left(\mathbb{R}^{3}, \boldsymbol{b}\right)$ is called a cuspidaledge if it is $\mathcal{A}$ equivalent to the germ $\left(u_{1}, u_{2}^{2}, u_{2}^{3}\right)$ (cf., Fig. 1) and a swallowtail if it is $\mathcal{A}$-equivalent to the germ $\left(3 u_{1}^{4}+u_{1}^{2} u_{2}, 4 u_{1}^{3}+2 u_{1} u_{2}, u_{2}\right)$ (cf., Fig.1).

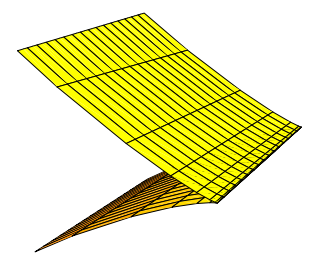

cuspidaledge

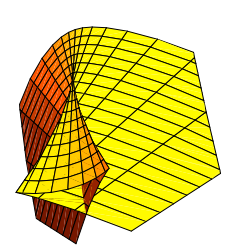

swallowtail

Fig. 1.

Following the terminology of Whitney [25], we say that a surface $\boldsymbol{X}: U \longrightarrow A d S^{4}$ has an excellent $S_{t}^{1} \times S_{s}^{2}$-nullcone Legendrian Gauss map $\mathbb{N}_{G}^{\sigma}$ if the Legendrian lift of $\mathbb{N}_{M}^{\sigma}$ is a stable Legendrian immersion at each point. In this case, the $S_{t}^{1} \times S_{s}^{2}$-nullcone Legendrian Gauss map $\mathbb{N} G_{M}^{\sigma}$ has only cuspidaledges and swallowtails as singularities. We have the following results analogous to the results of Banchoff et al [2].

Theorem 7.2 Let $\mathbb{N}_{G_{M}^{\sigma}}^{\sigma}:\left(U,\left(x_{0}, y_{0}\right)\right) \longrightarrow\left(A d S^{4}, p_{0}\right)$ be the excellent $S_{t}^{1} \times S_{s}^{2}$-nullcone Legendrian Gauss map of a spacelike surface $\boldsymbol{X}$ and $h_{v_{0}^{\sigma}}:\left(U,\left(x_{0}, y_{0}\right)\right) \longrightarrow \mathbb{R}$ be the nullcone height function germ at $\boldsymbol{v}_{0}^{ \pm}=\overline{\boldsymbol{e}_{1} \pm \boldsymbol{e}_{2}}\left(p_{0}\right)$, where $\sigma= \pm$. Then we have the following:

(1) $\left(x_{0}, y_{0}\right)$ is a lightlike parabolic point of $\boldsymbol{X}$ if and only if $\operatorname{L-corank}^{\sigma}\left(\boldsymbol{X},\left(x_{0}, y_{0}\right)\right)=1$.

(2) If $\left(x_{0}, y_{0}\right)$ is a lightlike parabolic point of $\boldsymbol{X}$, then $h_{v_{0}^{\sigma}}$ has the $A_{k}$-type singularity for $k=2,3$.

(3) Suppose that $\left(x_{0}, y_{0}\right)$ is a lightlike parabolic point of $\boldsymbol{X}$. Then the following conditions are equivalent:

(a) $\mathbb{N} \mathbb{G}_{M}^{\sigma}$ has a cuspidaledge at $\left(x_{0}, y_{0}\right)$

(b) $h_{v_{0}^{\sigma}}$ has the $A_{2}$-type singularity.

(c) $\operatorname{L-ord}{ }^{\sigma}\left(\boldsymbol{X},\left(x_{0}, y_{0}\right)\right)=2$.

(d) The tangent lightlike hyperbolic cylinder indicatrix is an ordinary cusp, where a curve $C \subset \mathbb{R}^{2}$ is called a ordinary cusp if it is diffeomorphic to the curve given by $\left\{\left(u_{1}, u_{2}\right) \mid u_{1}^{2}-u_{2}^{3}=\right.$ $0\}$.

(e) $\widehat{\mathbb{N G}}_{M}^{\sigma}$ has a fold at $\left(x_{0}, y_{0}\right)$.

(4) Suppose that $\left(x_{0}, y_{0}\right)$ is a lightlike parabolic point of $\boldsymbol{X}$. Then the following conditions are equivalent:

(a) $\mathbb{N} \mathbb{G}_{M}^{\sigma}$ has a swallowtail at $\left(x_{0}, y_{0}\right)$.

(b) $h_{v_{0}^{\sigma}}$ has the $A_{3}$-type singularity. 
(c) $\operatorname{L-ord}^{\sigma}\left(\boldsymbol{X},\left(x_{0}, y_{0}\right)\right)=3$.

(d) The tangent lightlike hyperbolic cylinder indicatrix is either a point, or a tachnodal, where a curve $C \subset \mathbb{R}^{2}$ is called a tachnodal if it is diffeomorphic to the curve given by $\left\{\left(u_{1}, u_{2}\right) \mid u_{1}^{2}-\right.$ $\left.u_{2}^{4}=0\right\}$.

(e) For each $\varepsilon>0$, there exist two distinct points $\left(x_{i}, y_{i}\right) \in U(i=1,2)$ such that

$$
\left\|\left(x_{0}, y_{0}\right)-\left(x_{i}, y_{i}\right)\right\|<\varepsilon
$$

for $i=1,2$, and the tangent lightlike hyperbolic cylinder to $M=\boldsymbol{X}(U)$ at $\left(x_{i}, y_{i}\right)$ coincide.

(f) $\widehat{\mathbb{N G}}_{M}^{\sigma}$ has a cusp at $\left(x_{0}, y_{0}\right)$.

Proof. We have shown that $\left(x_{0}, y_{0}\right)$ is a lightlike parabolic point if and only if

$$
\operatorname{L-corank}^{\sigma}\left(\boldsymbol{X},\left(x_{0}, y_{0}\right)\right) \geq 1 .
$$

We have $\operatorname{L-corank}^{\sigma}\left(\boldsymbol{X},\left(x_{0}, y_{0}\right)\right) \leq 2$. Since the nullcone height function germ $H:\left(U \times S_{t}^{1} \times S_{s}^{2},\left(\left(x_{0}, y_{0}\right), \boldsymbol{v}_{0}\right)\right) \longrightarrow \mathbb{R}$ can be considered as a generating family of the Legendrian lift of $\mathbb{N} \mathbb{G}_{M}^{\sigma}, h_{v_{0}^{\sigma}}$ has only the $A_{k^{-}}$-type singularities $(k=1,2,3)$. This means that the corank of the Hessian matrix of $h_{v_{0}^{\sigma}}$ at a lightlike parabolic point is 1 . The assertion (2) also follows. By the same reason, the conditions (3);(a),(b),(c) (respectively, (4); (a),(b),(c)) are equivalent. If the height function germ $h_{v_{0}^{\sigma}}$ has the $A_{2}$-type singularity, then it is $\mathcal{K}$-equivalent to the germ $\pm u_{1}^{2}+u_{2}^{3}$. Since the $\mathcal{K}$-equivalence preserve the diffeomorphism type of zero level sets, the tangent lightlike hyperbolic cylinder indicatrix is diffeomorphic to the curve given by $\pm u_{1}^{2}+u_{2}^{3}=0$. This is the ordinary cusp. The normal form for the $A_{3}$-type singularity is given by $\pm u_{1}^{2}+u_{2}^{4}$, so that the tangent lightlike hyperbolic cylinder indicatrix is diffeomorphic to the curve $\pm u_{1}^{2}+u_{2}^{4}=0$. This means that the condition (3),(d) (respectively, (4),(d)) is also equivalent to the other conditions.

Suppose that $\left(x_{0}, y_{0}\right)$ is a lightlike parabolic point, the singularities of the $S_{t}^{1} \times S_{s}^{2}$-nullcone Legendrian Gauss map are cuspidaledges or swallowtails. For the swallowtail point $\left(x_{0}, y_{0}\right)$, there is a self intersection curve (cf., Fig. 1) approaching to $\left(x_{0}, y_{0}\right)$. On this curve, there are two distinct points $\left(x_{i}, y_{i}\right)(i=1,2)$ such that $\mathbb{N} \mathbb{G}_{M}^{\sigma}\left(x_{1}, y_{1}\right)=\mathbb{N} \mathbb{G}_{M}^{\sigma}\left(x_{2}, y_{2}\right)$. By Lemma 5.2 , this means that the tangent lightlike hyperbolic cylinders to $M=\boldsymbol{X}(U)$ at $\left(x_{i}, y_{i}\right)$ coincide. Since there are no other singularities in this case, the condition $(4),(\mathrm{e})$ characterize a swallowtail point of $\mathbb{N} \mathbb{G}_{M}^{\sigma}$.

Finally, we know from the theory of Legendrian/Lagrangian singularities that a Legendrian map has a swallowtail (resp., cuspidaledge) if and only if the corresponding Lagrangian map has the cusp (resp., fold). This completes the proof.

\section{The Gauss-Bonnet type theorem}

In this section we give the definition of the global $S_{+}^{2}$-nullcone Gauss-Kronecker curvature and show a nullcone Gauss-Bonnet type theorem. Let $M$ be a closed orientable 2-dimensional manifold and $f: M \rightarrow A d S^{4}$ a spacelike embedding.

Since $A d S^{4}$ is time-oriented, we can globally choose future directions in the normal bundle $N(M)$ of $f(M)$. Let $\boldsymbol{e}_{1}$ be a timelike unit normal vector field along $f(M)$ directing towards the future direction at every point. We can now construct a spacelike unit normal vector field 
$\boldsymbol{e}_{2}$ as in $\S 2$. In this way we have a $S_{+}^{2}$-nullcone Lagrangian Gauss map globally defined on $M$, $\widehat{\mathbb{N G}}_{M}^{ \pm}: M \longrightarrow S_{+}^{2}$.

The global $S_{+}^{2}$-nullcone Gauss-Kronecker curvature function $\widehat{\mathcal{K}}_{\ell}^{ \pm}: M \rightarrow \mathbb{R}$ is then defined in the usual way in terms of the global $S_{+}^{2}$-nullcone Lagrangian Gauss map $\widehat{\mathbb{N G}}_{M}^{ \pm}$. By the equality (12), we have

$$
\widehat{\mathcal{K}}_{\ell}^{ \pm} d \mathfrak{a}_{M}=\left(\widehat{\mathbb{N G}}_{M}^{ \pm}\right)^{*} d \mathfrak{a}_{S_{+}^{2}},
$$

We can now prove the following Gauss-Bonnet type theorem.

Theorem 8.1 If $M$ is a closed orientable spacelike surface in $A d S^{4}$, then

$$
\int_{M} \widehat{\mathcal{K}}_{\ell}^{ \pm} d \mathfrak{a}_{M}=2 \pi \chi(M)
$$

where $d \mathfrak{a}_{M}$ is the area form and $\boldsymbol{\chi}(M)$ is the Euler number of $M$.

The principal idea of the proof is almost the same as that of the Gauss-Bonnet type theorem in [12]. Let $\pi_{3}^{5}: \mathbb{R}_{2}^{5} \longrightarrow \mathbb{R}^{3}$ be the canonical projection defined by $\pi_{3}^{5}\left(x_{1}, x_{2}, x_{3}, x_{4}, x_{5}\right)=$ $\left(0,0, x_{3}, x_{4}, x_{5}\right)$. Since $f(M)$ is a spacelike surface in $A d S^{4} \subset \mathbb{R}_{2}^{5}, \pi_{3}^{5}(f(M)$ is a smooth surface in the Euclidean space $\mathbb{R}^{3}$. Let $\mathbb{N}: M \longrightarrow S^{2}$ be the Euclidean Gauss map on $\pi_{3}^{5}(f(M))$. We show the following lemma.

Lemma 8.2 For a suitable choice of the direction $\mathbb{N}, \pi_{3}^{5} \circ \widehat{\mathbb{N G}}_{M}^{ \pm}$and $\mathbb{N}$ are homotopic.

Proof. Since $\mathbb{N G}_{M}^{ \pm}(p)$ is a lightlike vector, $\mathbb{N G}_{M}^{ \pm}(p) \notin \operatorname{Ker} \pi_{3}^{5}$. Moreover, $\operatorname{Ker} d \widehat{\tau}_{p} \subset \operatorname{Ker} \pi_{3}^{5}$, so that $\mathbb{N} \mathbb{G}_{M}^{ \pm}(p) \notin \operatorname{Ker} d \widehat{\tau}_{p}$. By definition, we can identify $d \widehat{\tau}_{p}\left(\mathbb{N G}_{M}^{ \pm}(p)\right)$ with $\widehat{\mathbb{N G}}_{M}^{ \pm}(p)$. Therefore, the fact $\mathbb{N G}_{M}^{ \pm}(p) \notin T_{p} f(M)$ induces that $\pi_{3}^{5} \circ \widehat{\mathbb{N G}}_{M}^{ \pm}(p) \notin T_{\pi_{3}^{5}(p)} \pi_{3}^{5}(f(M))$. This means that $\left\langle\pi_{3}^{5} \circ \widehat{\mathbb{N G}}_{M}^{ \pm}(p), \mathbb{N}(p)\right\rangle \neq 0$. We now choose the direction $\mathbb{N}$ such that $\left\langle\pi_{3}^{5} \circ \pi_{3}^{5} \circ \widehat{\mathbb{N G}}_{M}^{ \pm}(p), \mathbb{N}(p)\right\rangle \ngtr 0$.

On the other hand, we define a mapping $F: M \times[0,1] \longrightarrow S^{2}$ by

$$
F(p, t)=\frac{t \mathbb{N}(p)+(1-t) \pi_{3}^{5} \circ \widehat{\mathbb{N G}}_{M}^{ \pm}(p)}{\left\|t \mathbb{N}(p)+(1-t) \pi_{3}^{5} \circ \widehat{\mathbb{N G}}_{M}^{ \pm}(p)\right\|},
$$

where $\|\cdot\|$ is the Euclidean norm. If there exist $t^{\prime} \in[0,1]$ and $p^{\prime} \in M$ such that $t^{\prime} \mathbb{N}\left(p^{\prime}\right)+$ $\left(1-t^{\prime}\right) \pi_{3}^{5} \circ \widehat{\mathbb{N G}}_{M}^{ \pm}\left(p^{\prime}\right)=0$, then $\mathbb{N}\left(p^{\prime}\right)=-\pi_{3}^{5} \circ \widehat{\mathbb{N G}}_{M}^{ \pm}\left(p^{\prime}\right)$, contrary to the assumption that $\left\langle\pi_{3}^{5} \circ \widehat{\mathbb{N G}}_{M}^{ \pm}(p), \mathbb{N}(p)\right\rangle \ngtr 0$. Therefore $F$ is continuous and $F(p, 0)=\pi_{3}^{5} \circ \widehat{\mathbb{N G}}_{M}^{ \pm}(p)$ and $F(p, 1)=$ $\mathbb{N}(p)$.

It follows easily from the definitions of $\pi_{3}^{5}$ and $\widehat{\mathbb{N G}}_{M}^{ \pm}$that we may identify $\pi_{3}^{5} \circ \widehat{\mathbb{N G}}_{M}^{ \pm}$with $\widehat{\mathbb{N G}}_{M}^{ \pm}$. Since the mapping degree is a homotopy invariant, we obtain (cf., [8], Chapter 4, §9):

$$
\operatorname{deg} \widehat{\mathbb{N G}}_{M}^{ \pm}=\frac{1}{2} \chi(M) \text {. }
$$

Therefore we obtain

$$
\int_{M} \widehat{\mathcal{K}}_{\ell}^{ \pm} d \mathfrak{a}_{M}=\int_{M}\left(\widehat{\mathbb{N G}}_{M}^{ \pm}\right)^{*} d \mathfrak{a}_{S_{+}^{2}}=\operatorname{deg} \widehat{\mathbb{N G}}_{M}^{ \pm} \int_{S_{+}^{2}} d \mathfrak{a}_{S_{+}^{2}}=\operatorname{deg} \widehat{\mathbb{N G}}_{M}^{ \pm} \times 4 \pi=2 \pi \boldsymbol{\chi}(M) .
$$

This completes the proof of Theorem 8.1. 


\section{$9 \quad$ Horospherical Gauss maps}

In this section we consider the geometric meaning of the singularities of the hyperbolic Gauss map of $\widehat{M}=\widehat{\boldsymbol{X}}(U)$ in $H_{+}^{3}(-1)$, which was introduced in [12]. Here we denote that $\widehat{\boldsymbol{X}}=\widehat{\tau} \circ \boldsymbol{X}$ : $U \longrightarrow H_{+}^{3}(-1)$. The hyperbolic Gauss map of $\widehat{M}$ is defined as follows: Take a pseudo-orthogonal frame $\left\{\widehat{\boldsymbol{X}}, \widehat{\boldsymbol{E}}, \widehat{e}_{3}, \widehat{e}_{4}\right\}$ along $\widehat{M}$ in such a way that $\left\{\widehat{e}_{3}, \widehat{e}_{4}\right\}$ is a tangent frame. Then we have that $\widehat{\boldsymbol{X}} \pm \widehat{\boldsymbol{E}}$ is a normal lightlike vector and hence we can define a map $\mathbb{L}^{ \pm}: \widehat{M} \longrightarrow L C_{+}^{*}$ by $\mathbb{L}^{ \pm}(\hat{p})=\widehat{\boldsymbol{X}}(u) \pm \widehat{\boldsymbol{E}}(u)$ which is called the hyperbolic Gauss indicatrix (or the lightcone dual) of $\boldsymbol{X}$. The linear transformation $S_{\hat{p}}^{ \pm}=-d \mathbb{L}^{ \pm}(u): T_{\hat{p}} \widehat{M} \longrightarrow T_{\hat{p}} \widehat{M}$ is called the hyperbolic shape operator of $\widehat{M}=\widehat{\boldsymbol{X}}(U)$ at $\hat{p}=\widehat{\boldsymbol{X}}(u)$. The hyperbolic Gauss-Kronecker curvature of $\widehat{M}=\widehat{\boldsymbol{X}}(U)$ at $\hat{p}=\widehat{\boldsymbol{X}}(u)$ is defined to be $K_{h}^{ \pm}(u)=\operatorname{det} S_{\hat{p}}^{ \pm}$. If $\boldsymbol{x}=\left(x_{1}, x_{1}, x_{3}, x_{4}\right) \in \mathbb{R}_{1+}^{4}$ is a lightlike vector, then $x_{1} \neq 0$. Therefore we have $\tilde{\boldsymbol{x}}=\left(1, \frac{x_{2}}{x_{1}}, \frac{x_{3}}{x_{1}}, \frac{x_{4}}{x_{0}}\right) \in S_{+}^{2}$. The the hyperbolic Gauss map $\widetilde{\mathbb{L}^{ \pm}}: \widehat{M} \longrightarrow S_{+}^{2}$ on $\widehat{M}$ is defined by $\widetilde{\mathbb{L}}^{ \pm}(\hat{p})=\widetilde{\mathbb{L}^{ \pm}(\hat{p})}$. We remark that this definition of hyperbolic Gauss map is equivalent to the one introduced in [5, 7]. Let $T_{p} \widehat{M}$ be the tangent space of $\widehat{M}$ at $\hat{p}$ and $N_{\hat{p}} \widehat{M}$ be the pseudo-normal space of $T_{\hat{p}} \widehat{M}$ in $T_{\hat{p}} \mathbb{R}_{1}^{4}$. We have a linear transformation $\pi_{\hat{p}}^{t} \circ d \widetilde{\mathbb{L}}^{ \pm}(u): T_{\hat{p}} \widehat{M} \longrightarrow T_{\hat{p}} \widehat{M}$ by the identification of $U$ and $\widehat{\boldsymbol{X}}(U)=\widehat{M}$ via the embedding $\widehat{\boldsymbol{X}}$. We call the linear transformation $\widetilde{S}_{\hat{p}}^{ \pm}=-\pi_{\hat{p}}^{t} \circ d \widetilde{\mathbb{L}}^{ \pm}$the horospherical shape operator of $\widehat{M}$. The principal horospherical curvatures, $\widetilde{\kappa}_{i}^{ \pm}(\hat{p}), i=1,2$, are the eigenvalue of $\widetilde{S}_{\hat{p}}^{ \pm}$, the corresponding eigenvectors are called principal horospherical directions. These determine a couple of orthogonal line foliations on $\widehat{M}$, except at those points, called horoumbilics, for which both principal horospherical curvatures coincide. The horospherical principal configuration is composed by the principal horospherical curvature lines and the horoumbilic points. The horospherical Gauss-Kronecker curvature of $\widehat{M}$ is defined to be $\widetilde{K}_{h}^{ \pm}(\hat{p})=\operatorname{det} \widetilde{S}_{\hat{p}}^{ \pm}$.

We can use this setting in order to associate a hyperbolic Gauss map $\mathbb{H} \mathbb{G}_{M}^{ \pm}$to any spacelike surface $M \subset A d S^{4}$ by putting $\mathbb{H} \mathbb{G}_{M}^{ \pm}=\widetilde{\mathbb{L}}^{ \pm} \circ \widehat{\tau}: M \rightarrow S_{+}^{2}$. We observe that $\mathbb{H} \mathbb{G}^{ \pm}(p) \neq \widehat{\mathbb{N G}}_{M}^{ \pm}(p)$.

On the other hand, the intersection of a lightlike hyperplane $H(\boldsymbol{n}, c), \boldsymbol{n}=\left(0,1, n_{3}, n_{4}, n_{5}\right) \in$ $S_{+}^{2} \subset \mathbb{R}_{1+}^{4}$ and $c \in \mathbb{R}_{+}$, of $\mathbb{R}_{1+}^{4}$ with $H_{+}^{3}(-1)$ is a horosphere $h(\boldsymbol{n}, c)=\left\{\boldsymbol{x} \in H_{+}^{3}(-1) \mid-x_{2}+\right.$ $\left.n_{3} x_{3}+n_{4} x_{4}+n_{5} x_{5}=c\right\}$. The pull-back by $\widehat{\tau}$ of a horosphere $h(\boldsymbol{n}, c)$ in $H_{+}^{3}(-1)$ will be called round horosphere in $A d S^{4}$. We observe that $\widehat{\tau}^{1}(h(\boldsymbol{n}, c))=\left\{\left(\lambda \cos \theta, \lambda \sin \theta, x_{3}, x_{4}, x_{5}\right) \in\right.$ $\left.A d S^{4}: x_{3} n_{3}+x_{4} n_{4}+x_{5} n_{5}=c+\lambda\right\}$, is an invariant subset through the action of the group $S O(2) \times S O(3)$ over $\mathbb{R}_{2}^{5}$. Topologically, we can see it as a product $S^{1} \times h(\boldsymbol{n}, c)$ (with the radii of the fibers varying along the points of $h(\boldsymbol{n}, c))$. In [12] we observed that $\widetilde{\mathbb{L}}^{ \pm}$is a constant vector if and only if $\widehat{M}$ is a part of a horosphere in $H_{+}^{3}(-1)$. Therefore, $\mathbb{H} \mathbb{G}^{ \pm}$is constant if and only if $M$ is a subset of a round horosphere in $A d S^{4}$. Moreover,the pull-back by $\widehat{\tau}$ of the horospherical principal configuration on $\widehat{M}$ is, by definition, the horospherical principal configuration on $M$. We can interpret this as follows: For any $p \in M$, consider the lightlike vector $\mathbb{H}_{G}^{ \pm}(p)=\left(1, w_{3}, w_{4}, w_{5}\right)^{ \pm} \in S_{+}^{2} \subset \mathbb{R}_{1}^{4}$. Then $\widehat{\tau}^{-1}\left(\left(1, w_{3}, w_{4}, w_{5}\right)^{ \pm}\right)$is a circle $S_{t}^{1} \times\left\{\left(w_{3}, w_{4}, w_{5}\right)^{ \pm}\right\} \subset S_{t}^{1} \times S_{s}^{2}$. Consider now this circle in $T_{p} \mathbb{R}_{2 *}^{5} \equiv \mathbb{R}_{2}^{5}$. Then we have that the intersection $S_{t}^{1} \times\left\{\left(w_{3}, w_{4}, w_{5}\right)\right\} \times N_{p} M$ determines a normal lightlike direction $\ell^{ \pm}(p) \in S_{t}^{1} \times S_{s}^{2}$ for $M$ at $p$. In this way we obtain a lightlike normal field $\ell^{ \pm}$on $M$ that satisfies $\mathbb{H} \mathbb{G}^{ \pm}(p) \neq \widehat{\tau} \circ \ell^{ \pm}$. Moreover, if $S_{\ell^{ \pm}}(p)$ is the shape operator associated to the normal direction $\ell^{ \pm}(p)$, we have that $\widetilde{S}_{p}^{ \pm}=d_{p} \widehat{\tau} \circ S_{\ell^{ \pm}}(p)$. Therefore, the principal configuration associated to the normal field $\ell^{ \pm}$coincides with the horospherical principal configuration on $M$. The horospherical Gauss- 
Kronecker curvature of $M$ is defined as $\widetilde{K}_{h M}^{ \pm}(p)=\widetilde{K}_{h}^{ \pm}(\tau(p))$. We say that a point $p$ is a (positive or negative) horoparabolic point of $M$ if $\widetilde{K}_{h M}^{+}(p)=0$ or $\widetilde{K}_{h M}^{-}(p)=0$. Moreover, a point $p$ is said to be a horospherical point if it is both horoumbilic and horoparabolic. We observe that the horoflatness is invariant through motions in $S O(2) \times S O(3)$.

The Horospherical height functions family $\widetilde{H}: U \times S_{+}^{2} \longrightarrow \mathbb{R}$ on $M=\boldsymbol{X}(U) \subset A d S^{4}$ is defined by $\widetilde{H}(u, \boldsymbol{v})=\langle\widehat{\boldsymbol{X}}(u), \boldsymbol{v}\rangle$. We denote the Hessian matrix of the horospherical height function $\widetilde{h}_{v_{0}}(u)=\widetilde{H}\left(u, \boldsymbol{v}_{0}\right)$ at $u_{0}$ by $\operatorname{Hess}\left(\widetilde{h}_{v_{0}}\right)\left(u_{0}\right)$. The following are immediate consequences of ([12] Proposition 3.4 and Corollary 3.5).

Proposition 9.1 Let $\widetilde{H}: U \times S_{+}^{2} \longrightarrow \mathbb{R}$ be a lightcone height function on $\boldsymbol{X}: U \longrightarrow A d S^{4}$. Then $(u, \boldsymbol{v}) \in U \times S_{+}^{2}$ is a critical point of $\widetilde{H}$ if and only if $\boldsymbol{v}=\mathbb{H} \mathbb{G}^{ \pm}(u)$.

Moreover, provided $\boldsymbol{v}_{0}=\mathbb{H} \mathbb{G}^{ \pm}\left(u_{0}\right)$. Then we have

(1) $p=\boldsymbol{X}\left(u_{0}\right)$ is a horoparabolic point if and only if $\operatorname{det} \operatorname{Hess}\left(\widetilde{h}_{v_{0}}\right)\left(u_{0}\right)=0$.

(2) $p=\boldsymbol{X}\left(u_{0}\right)$ is a horospherical point if and only if $\operatorname{rank} \operatorname{Hess}\left(\widetilde{h}_{v_{0}}\right)\left(u_{0}\right)=0$.

Corollary 9.2 For a point $p=\boldsymbol{X}\left(u_{0}\right) \in M$, the following conditions are equivalent:

(1) The point $p \in M$ is a horoparabolic point (i.e., $\widetilde{K}_{h M}^{ \pm}(p)=0$ ).

(2) The point $p \in M$ is a singular point of the hyperbolic Gauss map $\mathbb{H} \mathbb{G}^{ \pm}$.

(3) $\operatorname{det} \operatorname{Hess}\left(\widetilde{h}_{v_{0}}\right)\left(u_{0}\right)=0$ for $\boldsymbol{v}_{0}=\mathbb{H} \mathbb{G}^{ \pm}\left(u_{0}\right)$.

We observe that the horospherical height functions family measures the contacts of the surface $M$ with round horospheres in $A d S^{4}$. The following result is also an immediate consequence of the above considerations.

Corollary 9.3 For $M \subset A d S^{4}$, the following conditions are equivalent:

(1) The horospherical Gauss-Kronecker curvature $\widetilde{K}_{h M}^{ \pm}$vanishes identically on $M$.

(2) The hyperbolic Gauss map $\mathbb{H} \mathbb{G}^{ \pm}$is constant over $M$.

(3) $M$ lies in a round horosphere.

We say that the embedding $\boldsymbol{X}$ is generic if its associated horospherical height functions family is structurally stable (see [14]), in other words, if $\widehat{X}$ is a generic embedding in $H_{+}^{3}(-1)$. The results obtained in [14] concerning the horospherical points of generically immersed surfaces in $H_{+}^{3}(-1)$ allow us to state:

Theorem 9.4 (1) The horospherical configurations in a neighbourhood of a horoumbilical point in a generic surface $M$ in $A d S^{4}$ are of Darbouxian type $D_{i}, i=1,2,3$. Therefore, the index of the horospherical principal direction fields at a horospherical point of a surface generically immersed in $A d S^{4}$ is $\pm \frac{1}{2}$.

(2) The number of horospherical points of any closed surface $M$ generically immersed in $A d S^{4}$ is greater or equal than $2|\chi(M)|$, where $\chi(M)$ denotes the Euler number of $M$.

(3) Any 2-sphere generically immersed in $A d S^{4}$ has at least 4 horoumbilical points.

On the other hand, as seen in [12], when $\widehat{M}$ is a closed orientable surface in $H_{+}^{3}(-1)$, we can consider a globally defined hyperbolic Gauss map on $\widehat{M}$ (and thus on $M$ ), and consequently, a globally defined Gauss-Kronecker curvature function on $\widehat{M}$ (and thus on $M$ ). For the purposes 
of the following result, we can either fix the superindex + or - in the above arguments and denote by $\widetilde{\mathcal{K}}_{h M}$ the globally defined Gauss-Kronecker curvature function on $M$ and by $\widetilde{\mathcal{K}}_{h}$ the globally defined Gauss-Kronecker curvature function on $\widehat{M}$. We first observe that if $d \mathfrak{v}_{M}$ and $d \mathfrak{v}_{\widehat{M}}$ represent respectively the volume forms of $M$ and $\widehat{M}$. If $M$ is a closed orientable spacelike surface in $A d S^{4}$, then

$$
\int_{M} \widetilde{\mathcal{K}}_{h M} d \mathfrak{a}_{M}=\int_{\widehat{M}} \widetilde{\mathcal{K}}_{h} d \mathfrak{a}_{\widehat{M}}
$$

Then, since $\widehat{\tau}$ determines a diffeomorphism between $M$ and $\widehat{M}$, we have that their Euler characteristics coincide. Therefore, as a consequence of the Gauss-Bonnet Theorem obtained in [12] for the closed orientable surfaces immersed in Hyperbolic 3-space, we can state:

Theorem 9.5 If $M$ is a closed orientable spacelike surface in $A d S^{4}$, then

$$
\int_{M} \widetilde{\mathcal{K}}_{h M} d \mathfrak{a}_{M}=2 \pi \chi(M)
$$

where $d \mathfrak{a}_{M}$ is the area form and $\chi(M)$ is the Euler number of $M$.

\section{References}

[1] V. I. Arnol'd, S. M. Gusein-Zade and A. N. Varchenko, Singularities of Differentiable Maps, vol. I, Birkhäuser. (1986)

[2] T. Banchoff, T. Gaffney and C. McCrory, Cusps of Gauss Mappings, Research Notes in Mathematics 55 Pitman, London. (1982)

[3] Th. Bröcker, Differential Germs and Catastrophes. Mathematical Society Lecture Note Series, 17. Cambridge University Press, Cambridge-New York (1975).

[4] J. W. Bruce and P. J. Giblin, Curves and singularities (second edition), Cambridge University press. (1992)

[5] R. L. Bryant, Surfaces of mean curvature one in hyperbolic space in Théorie des variétés minimales et applications (Palaiseau, 1983-1984), Astérisque No. 154-155 (1987), 12, 321347,353 (1988).

[6] S. Chandrasekhar, The Mathematical Theory of Black Holes, International Series of Monographs on Physics. 69 Oxford Univeristy press, 1983.

[7] C. L. Epstein, The hyperbolic Gauss map and quasiconformal reflections. J. Reine Angew. Math., 372 (1986), 96-135

[8] V. Guillemin and A. Pollack, Differential Topology, Prentice-Hall (1974).

[9] S. Izumiya, D. Pei and T. Sano, Singularities of hyperbolic Gauss maps, Proceedings of London Mathematical Society, (3) 86 (2003), 485-512.

[10] S. Izumiya, D. Pei and M. C. Romero Fuster, The lightcone Gauss map of a spacelike surfaces in Minkowski 4-space, Asian Journal of Mathematics, 8 (2004), 511-530.

[11] S. Izumiya, M. Kossowski, D. Pei and M. C. Romero Fuster, Singularities of lightlike hypersurfaces in Minkowski 4-space, Tohoku Mathematical Journal, 58 (2006), 71-88. 
[12] S. Izumiya and M.C. Romero-Fuster, The horospherical Gauss-Bonnet type theorem in hyperbolic space. J. Math. Soc. Japan 58 (2006), 965-984.

[13] S. Izumiya and M.C. Romero Fuster, The lightlike flat geometry on spacelike submanifolds of codimension two in Minkowski space. Selecta Math. (N.S.) 13 (2007), no. 1, 23-55.

[14] S. Izumiya, J.J. Nunño Ballesteros and M.C. Romero Fuster, Global properties of codimension two spacelike submanifolds in Minkowski space. To appear in Advances in Geometry.

[15] M. Kossowski, The $S^{2}$-valued Gauss maps and split total curvature of space-like codimension-2 surface in Minkowski space, J. London Math. Soc.(2) 40 (1989), 179-192.

[16] M. Kossowski, The intrinsic conformal structure and Gauss map of a light-like hypersurface in Minkowski space, Transactions of the American mathematical society, 316 Number 1, (1989), 369-383.

[17] J. A. Little, On singularities of submanifolds of high dimensional Euclidean space, Annali Mat.Pura et Appl.(ser.4A), 83 (1969), 261-336.

[18] J. Martinet, Singularities of Smooth Functions and Maps, London Math. Soc. Lecture Note Series, Cambridge Univ. Press, 58. (1982)

[19] J. N. Mather, Stability of $C^{\infty}$-mappings IV:Classification of stable germs by $\mathbb{R}$ algebras, Publi. Math. I.H.E.S., 37 (1970), 223-248.

[20] C. W. Misner, K. S. Thorne and J. W. Wheeler, Gravitation, W. H. Freeman and Co., San Francisco, CA (1973).

[21] J. A. Montaldi, On contact between submanifolds, Michigan Math. J., 33 (1986), 81-85.

[22] J. A. Montaldi, On generic composites of maps. Bull. London Math. Soc., 23 (1991), 81-85.

[23] B. O’Neill, Semi-Riemannian Geometry, Academic Press, New York. (1983)

[24] G. Wassermann, Stability of Caustics, Math. Ann., 2210 (1975), 443-50.

[25] H. Whitney, On singularities of mappings of Euclidean spaces I, Ann. of Math., 62 (1955), $374-410$.

[26] V. M. Zakalyukin, Lagrangian and Legendrian singularities. Funct. Anal. Appl., 10 (1976), $23-31$.

Shyuichi Izumiya, Department of Mathematics, Hokkaido University, Sapporo 060-0810,Japan e-mail:izumiya@math.sci.hokudai.ac.jp

Donghe Pei, School of Mathematics and Statistics, Northeast Normal University, Changchun 130024, P.R.China

e-mail:peidh340@nenu.edu.cn

María del Carmen Romero Fuster, Departament de Geometría i Topología, Universitat de València, 46100 Burjassot (València), Espanya

e-mail:carmen.romero@post.uv.es 